\title{
Complexity growth rate during phase transitions
}

\author{
Mahdis Ghodrati ${ }^{*}$ \\ Center for Gravitation and Cosmology, College of Physical Science and Technology, \\ Yangzhou University, Yangzhou 225009, China
}

(Received 30 August 2018; revised manuscript received 16 October 2018; published 12 November 2018)

\begin{abstract}
We present evidence for the connection between the potential of different fields and complexity growth rates both in conformal and confining cases. By studying different models, we also establish a strong connection between phase transitions and the discontinuities in the complexity growth rates. In the first example, for dyonic black holes that are dual to van der Waals fluids, we find a similar first-order phase transition in the behavior of the complexity growth rate. We then compare the Schwinger effect and also the behavior of complexity in the anti-de Sitter (AdS) and AdS soliton backgrounds and comment on the connection between them. Finally, in a general Gubser model of QCD, we present the connections between the potentials, entropies, speeds of sound, and complexity growth rates during crossover and first- and second-order phase transitions, and also the behavior of quasinormal modes.
\end{abstract}

DOI: 10.1103/PhysRevD.98.106011

\section{INTRODUCTION}

In addition to the Ryu-Takayanagi formula [1]which connects the entanglement entropy as a quantuminformation quantity in the boundary and the area of a codimension-two hypersurface as a geometric quantity in the bulk-holographic quantum complexity [2-4] has recently been proposed as another connection between quantum information and geometry.

On the quantum-information side, complexity would be the number of gates needed to go from one specific quantum state to another, and thus it quantifies how difficult a computational task would be. On the geometrical side, it could be calculated from the volume of a codimensionone surface which extends between two boundaries (complexity = volume; the $\mathrm{CV}$ conjecture) or the action on the Wheeler-DeWitt patch (complexity = action; the CA conjecture) $[3,5]$. These conjectures have been studied extensively; see Refs. [6-26] for some examples.

In order to make this new connection more precise and use it in the holographic context, a more exact definition of complexity for field theories is needed. The initial attempts were for free scalar fields [27,28], simple fermionic fields [29-31], and recently coherent [32] and simple interacting quantum field theories [33]. Generally,

\footnotetext{
*mahdisg@yzu.edu.cn
}

Published by the American Physical Society under the terms of the Creative Commons Attribution 4.0 International license. Further distribution of this work must maintain attribution to the author(s) and the published article's title, journal citation, and DOI. Funded by SCOAP. there are some ambiguities in defining the specific gates needed for each case.

In this work, we first examine if a relationship exists between quantum fluctuations of the system in different models (for instance, between particle pair-creation and annihilation rates) and the rates of growth of complexity. There are actually several reasons one might conjecture that such a connection between them exists. One reason is that the only physical processes that would still occur after thermal equilibrium are quantum fluctuations, which could be the main source of complexity growth. Also, it has been found that the complexity growth rate of black holes and also the world sheet of a string dual to an entangled particleantiparticle would both saturate the Lyapunov bound [34]. Also, generally increasing the energy of the system would increase the complexity growth rate.

To further examine this idea, in Sec. II we study the potential wells and barriers of different fields in different models and their complexity growth rates, and in fact we observe such a relationship.

To learn more about the nature of holographic complexity, one could study its behavior in more exotic cases [35-43] such as various phase transitions and then try to establish the connections with other physical quantities of the system. This quest is the main purpose of this work.

In Ref. [36], the complexity in an interesting QCD model was studied, and similarities between the first, second, and crossover phase transitions in both the entropy and complexity growth rate were observed. Therefore, in that work it was shown that complexity could in fact act as a probe of different phase transitions, particularly confinement. Also, in Ref. [40], using models of quantum harmonic oscillators, it was shown that complexity can act as a probe of quantum 
quenches and can even capture features that entanglement entropy is not capable of probing. Along the lines of those papers, we would like to study the complexity growth rates in various phase transition setups.

Therefore, in Sec. III A we first study the charged dyonic black hole model which is the system that could mimic the properties of a van der Waals fluid, and-using the Gibbs free energy-we observe the first- and second-order phase transitions. Using the full time-dependent complexity growth rate, we also observe a phase transition in the complexity growth rate of this model.

Then, in Sec. III B we consider anti-de Sitter (AdS) and AdS soliton backgrounds, study their potentials and the complexity growth rate in each background, and examine the connection between the phase transitions (such as tachyon condensation and complexity growth rate behaviors), specifically in the IR and UV regions. We also compare the Schwinger effect phase diagrams in these two cases and comment on their relationship with complexity.

Finally, in Sec. IV we study the phase transitions in the Gubser model of QCD. In this model, by fine-tuning the parameters of the confining potential in the diagram of entropy versus temperature, one can obtain crossover, first-, and second-order phase transitions for $V_{\mathrm{QCD}}, V_{1}$, and $V_{2}$, respectively. Also, we study another model of improved holographic QCD, $V_{\mathrm{IHQCD}}$, which could present a firstorder phase transition while providing a more effective model for the dynamical properties of QCD, such as asymptotic freedom and a more realistic bulk viscosity. This model shows substantial differences in the behavior of quasinormal modes and complexity, which we will discuss in detail.

Therefore, we study the behavior of entropy, the complexity growth rate, the speed of sound, and the potentials for all four of these models and compare their behavior at lower temperatures and specifically near the phase transitions. We also outline the relationship between the crossover from hydrodynamic to nonhydrodynamic modes of each model, and the resulting topological jump in the complexity growth rate around the phase transition point.

Finally, we conclude with a discussion in Sec. V.

\section{THE RELATIONSHIP BETWEEN PHYSICAL PARAMETERS AND COMPLEXITY GROWTH RATES}

By comparing the results for the complexity growth rates in different black hole solutions with different fields, in this section we study the connections between the action growth rates and mass, charges with different physical properties, and their coupling to gravity. Specifically, we study solutions with dilaton, Maxwell, and phantom fields (which have ghosts) and compare their behaviors.

In this section, we first review the already calculated results for different backgrounds which have fields with different natures, and then we discuss the connection between the complexity growth rate and the parameters of the solution and the physical interpretations.

\section{A. Charged dilaton black hole in AdS space}

First, we consider the Einstein-Maxwell-dilaton model [6]

$S=\frac{1}{16 \pi} \int d^{4} x \sqrt{-g}\left(R-2(\partial \phi)^{2}-V(\phi)-e^{-2 \phi} F^{2}\right)$,

where the potential $V(\phi)$, shown in Fig. 1, for the dilaton field is

$$
V(\phi)=-\frac{4}{l^{2}}-\frac{1}{l^{2}}\left[e^{2\left(\phi-\phi_{0}\right)}+e^{-2\left(\phi-\phi_{0}\right)}\right] .
$$

Here $\phi_{0}$ is a constant and $l$ is the AdS radius. So, this potential is the combination of a constant value and two Liouville-type potentials. The spherically symmetric charged dilaton black hole solution of this action would be

$$
\begin{gathered}
d s^{2}=-f(r) d t^{2}+f^{-1}(r) d r^{2}+U^{2}(r) d \Omega^{2}, \\
F_{r t}=\frac{Q e^{2 \phi}}{U^{2}}, \quad e^{2 \phi}=e^{2 \phi_{0}}\left(1-\frac{2 D}{r}\right),
\end{gathered}
$$

where

$$
\begin{aligned}
f(r) & =1-\frac{2 M}{r}+\frac{r(r-2 D)}{l^{2}}, \quad U^{2}(r)=r(r-2 D), \\
D & =\frac{Q^{2} e^{2 \phi_{0}}}{2 M},
\end{aligned}
$$

where $D$ is the dilaton charge and $\phi_{0}$ is the asymptotic constant value of the dilaton [44]. Note that when $\phi=\phi_{0}=0$, this solution reduces to the ReissnerNordström black hole.

In Ref. [6], for the charged dilaton AdS black hole, the complexity growth rate at late times was found to be

charged dilation AdS BH:

$$
\dot{C} \sim \frac{d S}{d t}=2 M-Q^{2} e^{2 \phi_{0}}\left(\frac{1}{2 M}+\frac{1}{r_{+}}\right),
$$

where, as one expects, for the case of $Q=0$ the result reduces to that for the AdS-Schwarzschild solution. Also, note that this black hole has only one horizon $r_{+}$, which is a function of $M, Q$, and $l$. It could be found simply by using $\left.f(r)\right|_{r=r_{+}}=0$, but as it is a long relation we do not display it here.

We want to study the effect of each parameter on the complexity growth rate while other parameters are fixed, and find a physical explanation for the specific behaviors.

First, we keep $M$ and $Q$ constant and only study the effect of the boundary dilaton field $\phi_{0}$ on the complexity 


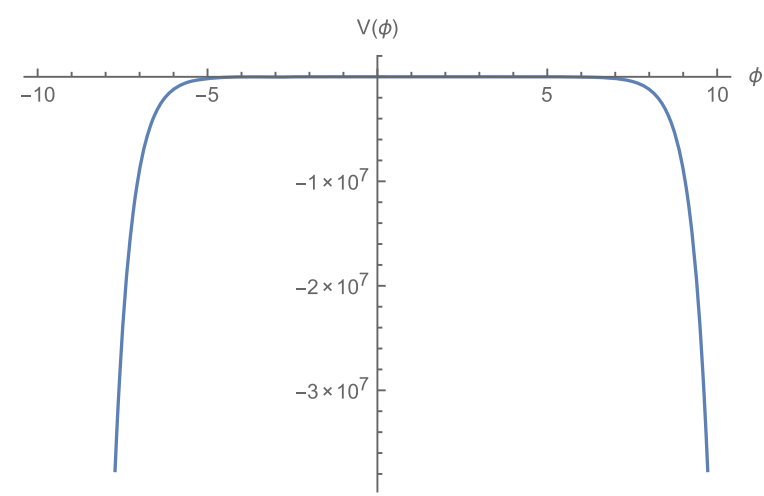

FIG. 1. Two Liouville-type potentials (2.2), for $l=\phi_{0}=1$.

growth rate. The plot is shown in Fig. 2(a). (In all cases we take $l=1$ ).

From Fig. 2(a) one can notice that for all of the negative and small positive values of $\phi_{0}$, the complexity is constant. Then, at a specific positive value of $\phi_{0}$, it decreases exponentially. Therefore, as a first observation one could generally say that increasing the dilaton field decreases the complexity growth rate. The specific value of $\phi_{0}$ at which this sudden decrease would happen would decrease by increasing $Q$, but it does not change much by varying $M$. However, increasing $M$ would greatly increase the initial value of $\dot{C}$ for negative dilaton fields.

Recently, in Ref. [45] the effect of a dilaton field on the complexity growth rate was studied further, and the full time behaviors of the action growth rate for the AdS dilaton black hole and asymptotically Lifshitz black holes were considered. Generally, as it was shown in Ref. [45] using another method, the dilaton field should actually decrease the growth rate of complexity, which we observe here as well. In Sec. IV, the same result is seen in models of confining potentials, so we conjecture that this is a universal effect.

The physical reason behind this could be explained by noting that in string theory, the dilaton field actually describes how strongly open strings couple to one another. In fact, in perturbation theory, the coupling constant of open strings is proportional to the exponential value of the dilaton's expectation value, $g_{s}=e^{\langle\phi\rangle}$. So the more that the strings are coupled to each other, the more difficult it would be to go from one state to another, and the rate of computation and complexification would be lower.

In Fig. 2(c), the relationship between the complexity growth rate and mass is shown. The explanation for this relationship is much simpler: for bigger masses there are more degrees of freedom and a correspondingly higher number of gates in the dual boundary, and therefore the rate of complexity growth is higher. In fact, the growth is linear which intuitively makes sense as the mass and number of gates are linearly connected.

The behavior of the complexity growth rate with respect to charge [Fig. 2(b)] can also be explained by considering the relation between the complexity growth rate and the height of potential barriers. Note that for the case of a Schwarzschild black hole, which has no charge, the height of the potential barrier is just equal to the temperature, while for the case of charged black holes, such as a Reissner-Nordström (RN) black hole, this barrier (due to its charge) is much higher [46], which would decrease the rate of complexity growth.

In Ref. [46], the scrambling behavior in the field of a "near-extremal charged black hole" was studied. There it was noted that due to the buildup of momentum of a particle accelerating through the long throat of the Reissner-Nordström geometry, some degrees of freedom become decoupled and cannot enter in the process of computation and complexification. The greater the charge of the black hole, the longer the throat of a RN black hole would be, which causes more degrees of freedom to decouple and therefore the complexity growth rate would decrease.

Also, note that a charge in the bulk would be dual to a current in the boundary field theory. A larger black hole charge would be dual to higher current with higher momentum. The larger momentum of the current would actually decrease the complexification rate.

The dependence of the complexity growth rate on two parameters of a black hole (while keeping the third one

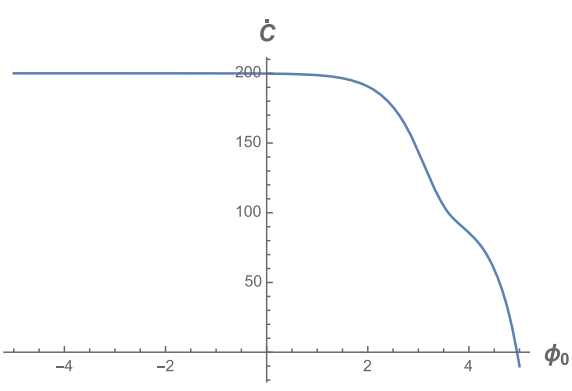

(a) $M=100, Q=1$

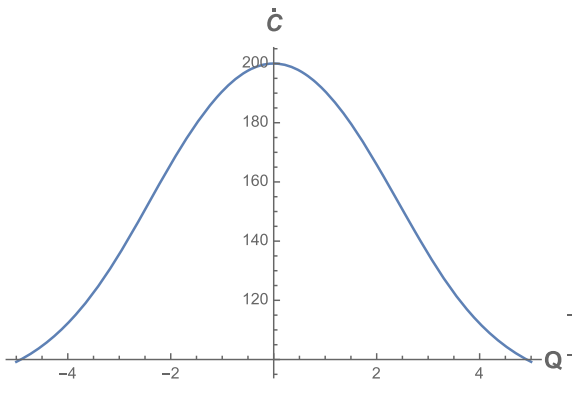

(b) $M=100, \phi_{0}=2$

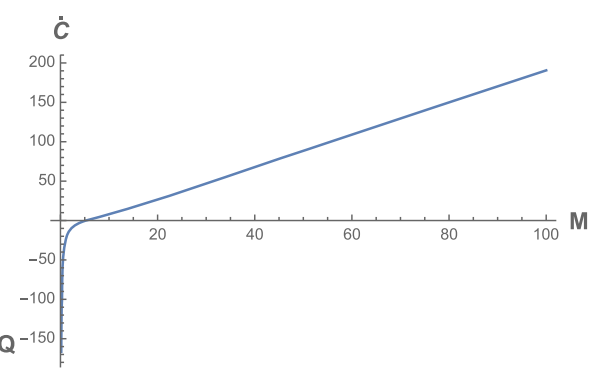

(c) $Q=1, \phi_{0}=2$

FIG. 2. The dependence of the complexity growth rate on each parameter of the charged dilaton black hole. 


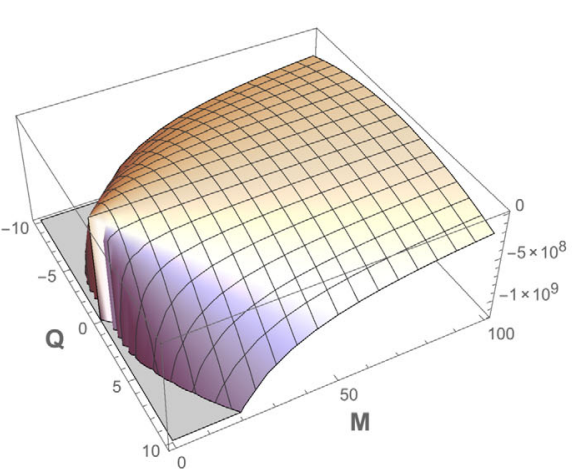

(a) $\phi_{0}=10$

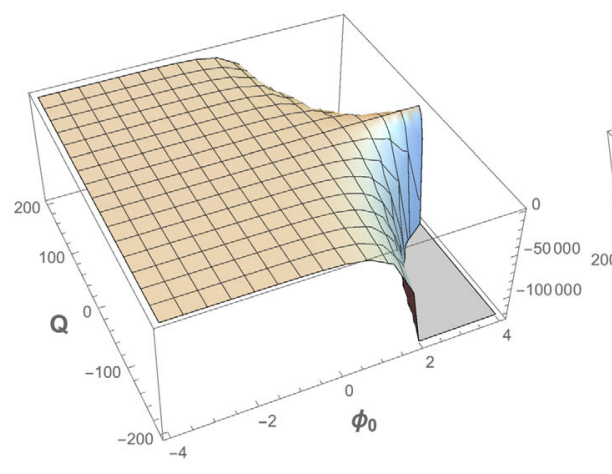

(b) $M=10$

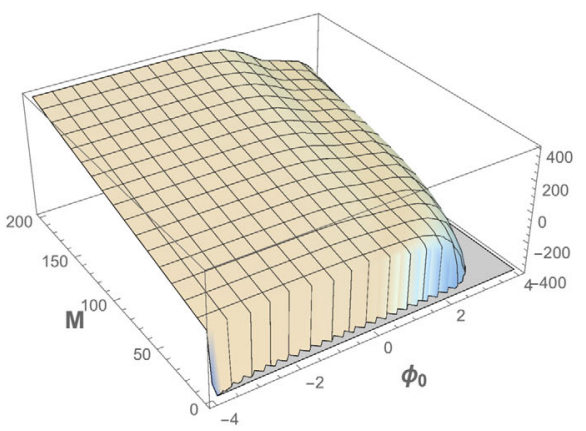

(c) $Q=5$

FIG. 3. The dependence of the complexity growth rate on two parameters of a charged dilaton black hole.

constant) is shown in Fig. 3. In Fig. 3(a) we see that for higher masses a changing charge does not drastically change the complexity growth rate, while for lower masses an increasing charge would dramatically decrease the growth rate.

In Fig. 3(b) we see that when the dilaton field is negative or very small and close to zero, even for higher charges, the complexity growth rate does not change. This is because for small and negative $\phi_{0}$ the string couplings would be very weak, and therefore even for high charges the complexity growth rate would not change. On the other hand, increasing the dilaton would increase the couplings between string modes, which would subsequently increase the dependence of the growth rate on the charge.

In Fig. 3(c), we again see that for a negative dilaton field the complexity growth rate is constant, and even increasing the mass $M$ cannot change this flat behavior. However, increasing the mass at each $\phi_{0}$ would linearly increase the complexity growth rate. Also, note that for larger masses an increasing dilaton field causes a milder decrease of the complexity growth rate.

\section{B. Born-Infeld black hole in AdS Space}

Another example of a charged black hole in AdS is a Born-Infeld black hole, which has the action

$S=\frac{1}{16 \pi} \int d^{4} x \sqrt{-g}\left[R+\frac{6}{l^{2}}+4 \beta^{2}\left(1-\sqrt{1+\frac{F_{\mu \nu} F^{\mu \nu}}{2 \beta^{2}}}\right)\right]$,

where one can get a static spherically symmetric black hole solution as follows:

$$
\begin{aligned}
d s^{2} & =-f(r) d t^{2}+f^{-1}(r) d r^{2}+r^{2} d \Omega^{2}, \\
F_{r t} & =\frac{Q}{\sqrt{r^{4}+Q^{2} / \beta^{2}}},
\end{aligned}
$$

where

$$
\begin{aligned}
f(r)= & 1-\frac{2 M}{r}+\frac{r^{2}}{l^{2}}+\frac{2 \beta^{2}}{r} \int_{r}^{\infty} d x\left(\sqrt{x^{4}+Q^{2} / \beta^{2}}-x^{2}\right) \\
= & 1-\frac{2 M}{r}+\frac{r^{2}}{l^{2}} \\
& +\frac{2 \beta^{2}}{r}\left[I\left(r=\infty ; a^{2}=\frac{Q^{2}}{\beta^{2}}-I\left(r ; a^{2}=\frac{Q^{2}}{\beta^{2}}\right)\right],\right.
\end{aligned}
$$

and $I\left(r ; a^{2}\right) \equiv \int^{r} d x\left(\sqrt{x^{4}+a^{2}}-x^{2}\right)$.

Here, $M$ and $Q$ are the mass and charge of the black hole. Also note that when $\beta \rightarrow \infty$, the Born-Infeld theory reduces to the Maxwell theory with $\mathcal{L}(F)=-F^{2}$. The chemical potential of this black hole is

$$
\mu(r) Q=\frac{Q^{2}}{r}{ }_{2} F_{1}\left[\frac{1}{4}, \frac{1}{2}, \frac{5}{4},-\frac{Q^{2}}{\beta^{2} r^{4}}\right] .
$$

As found in Ref. [6], when $\beta^{2} Q^{2} \geq 1 / 4$ the black hole solution has two horizons, and when $\beta^{2} Q^{2}<1 / 4$ it only has one horizon with the inner one being absent. So changing the coupling $\beta$ or the charge $Q$ could greatly impact the complexity as it significantly changes the geometry.

For the case with two horizons, Ref. [6] found the action growth as

$\frac{d S}{d t}=\left.\left(\frac{Q^{2}}{r}{ }_{2} F_{1}\left[\frac{1}{4}, \frac{1}{2}, \frac{5}{4},-\frac{Q^{2}}{\beta^{2} r^{4}}\right]\right)\right|_{r_{+}} ^{r_{-}}=\mu_{-} Q-\mu_{+} Q$,

and for the case with one horizon, it is

$$
\frac{d S}{d t}=2 M-\frac{Q^{2}}{r_{+}}{ }_{2} F_{1}\left[\frac{1}{4}, \frac{1}{2}, \frac{5}{4},-\frac{Q^{2}}{\beta^{2} r_{+}^{4}}\right]-\beta^{\frac{1}{2}} Q^{\frac{3}{2}} \frac{\Gamma\left(\frac{1}{4}\right) \Gamma\left(\frac{5}{4}\right)}{3 \Gamma\left(\frac{1}{2}\right)},
$$




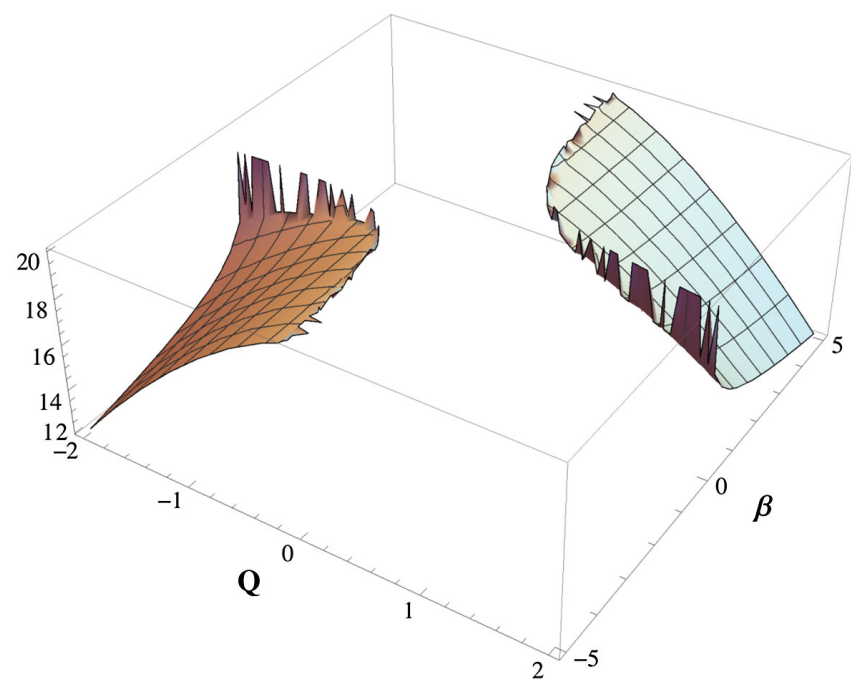

FIG. 4. Complexity growth rate for the case of a black hole with one horizon in Born-Infeld theory, with $M=10$ and $l=1$.

where

$$
\begin{aligned}
r_{+}= & \frac{l^{2}}{12 \beta^{2} l^{2}+9}\left(-3-2 \beta^{2} l^{2}\right. \\
& \left.+2 \sqrt{\beta^{4} l^{4}+3 \beta^{2} Q^{2}\left(4 \beta^{2} l^{2}+3\right)}\right)
\end{aligned}
$$

Again, one can see that the relationship with respect to mass $M$ is linearly increasing. From Fig. 4 one can see that in this case (similar to the previous case) increasing the charge $Q$ would decrease the complexity growth rate, while increasing the coupling constant $\beta$ (which couples the gauge field $F_{\mu \nu}$ to the metric $g$ ) would decrease the complexity growth rate.

Note that due to a strong singularity in this case, at low charges, low temperatures, or low $\beta$ the complexity growth would not be smooth, which can be seen from the plot.

\section{Charged black hole with a phantom Maxwell field}

As another example, we consider the action of Einsteinphantom-Maxwell theory with a negative cosmological constant,

$$
S=\frac{1}{16 \pi} \int d^{4} x \sqrt{-g}\left(R+\frac{6}{l^{2}}+F_{\mu \nu} F^{\mu \nu}\right),
$$

whose solution is

$$
\begin{aligned}
& d s^{2}=-f(r) d t^{2}+f^{-1}(r) d r^{2}+r^{2} d \Omega^{2}, \\
& F_{t r}=\frac{Q}{r^{2}}, \quad f(r)=1+\frac{r^{2}}{l^{2}}-\frac{2 M}{r}-\frac{Q^{2}}{r^{2}} .
\end{aligned}
$$

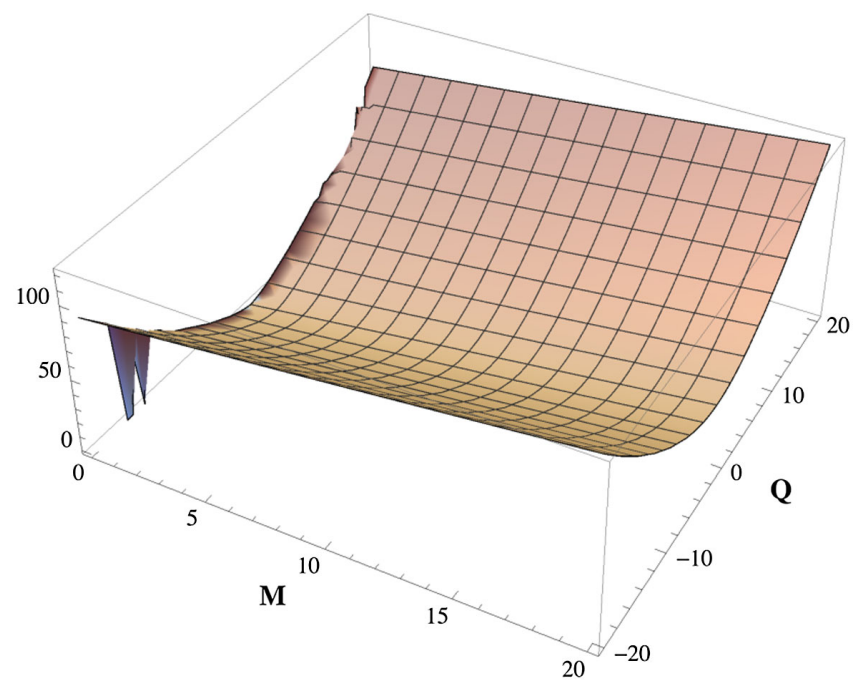

FIG. 5. Complexity growth rate for the case of a phantom Maxwell field with a ghost.

Note that in this case the Maxwell term has the wrong sign. Its Penrose diagram is the same as that of an AdS Schwarzschild black hole. The action growth rate is $\frac{d S}{d t}=2 M+\mu_{+} Q$.

From Fig. 5, one can see that increasing $M$ would linearly increase the complexity growth rate; however, unlike previous cases, increasing the charge $Q$ would increase the complexity growth rate.

Note that the phantom Maxwell field is different from other cases in several significant ways. As mentioned before, the Maxwell term has the wrong sign. Also, other cases satisfy the Lloyd bound at late times but this case violates it, even at late times; this is because the phantom field is actually a "ghost field" which violates the strong energy condition. So generally one could say that the charges that violate the strong energy condition (such as ghosts) would increase the complexity growth rate, note Fig. 6.

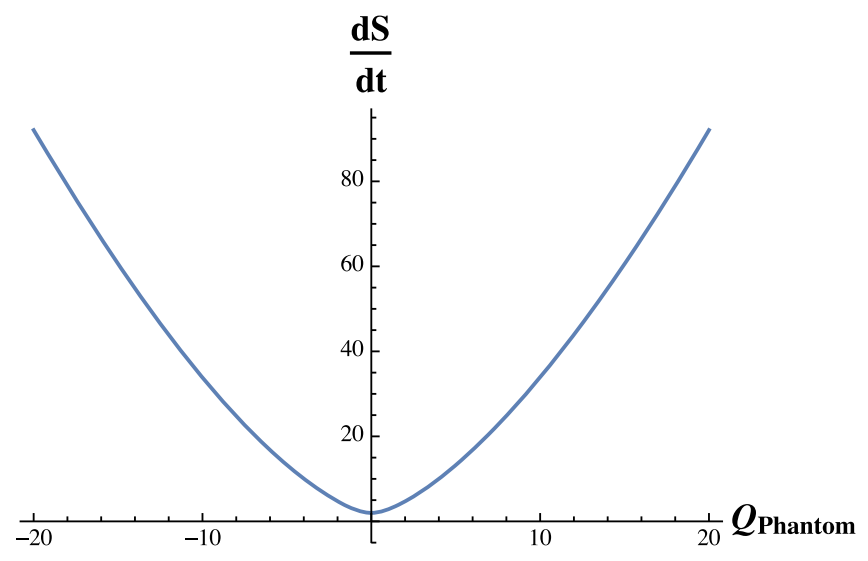

FIG. 6. The behavior of the complexity growth rate for the case of a phantom Maxwell field (ghost) is opposite that of other cases, such as in Fig. 2(b) which has physical charges. 


\section{PHASE TRANSITIONS AND THE COMPLEXITY GROWTH RATE WITH A CHARGE OR KINK}

We now study the full time behaviors of the complexity growth rates for the dyonic and AdS soliton black holes and compare them with their different phase transitions. We find that generally the complexity growth rate could probe any kind of phase transition and instability.

\section{A. Complexity in dyonic black holes}

First, we consider the dyonic black hole in the holographic setup similar to Ref. [47]. We chose this type of black hole as it has both electric and magnetic charges. It could be a solution to the Reissner-Nordström action with a negative cosmological constant,

$$
I=\frac{1}{16 \pi} \int d^{d} \sqrt{g}\left[\mathcal{R}-2 \Lambda-F^{\mu \nu} F_{\mu \nu}\right]
$$

where the static spherically symmetric solution is

$$
\begin{aligned}
d s^{2} & =-f(r) d t^{2}+\frac{d r^{2}}{f(r)}+r^{2} d \Omega^{2}, \\
f(r) & =1-\frac{\Lambda r^{2}}{3}-\frac{2 m}{r}+\frac{q_{E}^{2}+q_{M}^{2}}{r^{2}}, \\
A & =\left(-\frac{q_{E}}{r}+\frac{q_{E}}{r_{+}}\right) d t+\left(q_{M} \cos \theta\right) d \phi .
\end{aligned}
$$

The charge and mass of this solution are

$$
\begin{aligned}
M & =\frac{2 \Omega_{2}}{16 \pi}\left(r_{+}+r_{-}+\frac{1}{l^{2}} \frac{r_{+}^{4}-r_{-}^{4}}{r_{+}-r_{-}}\right), \\
Q^{2} & =\frac{2 \Omega_{2}}{8 \pi} r_{+} r_{-}\left(1+\frac{1}{l^{2}} \frac{r_{+}^{3}-r_{-}^{3}}{r_{+}-r_{-}}\right),
\end{aligned}
$$

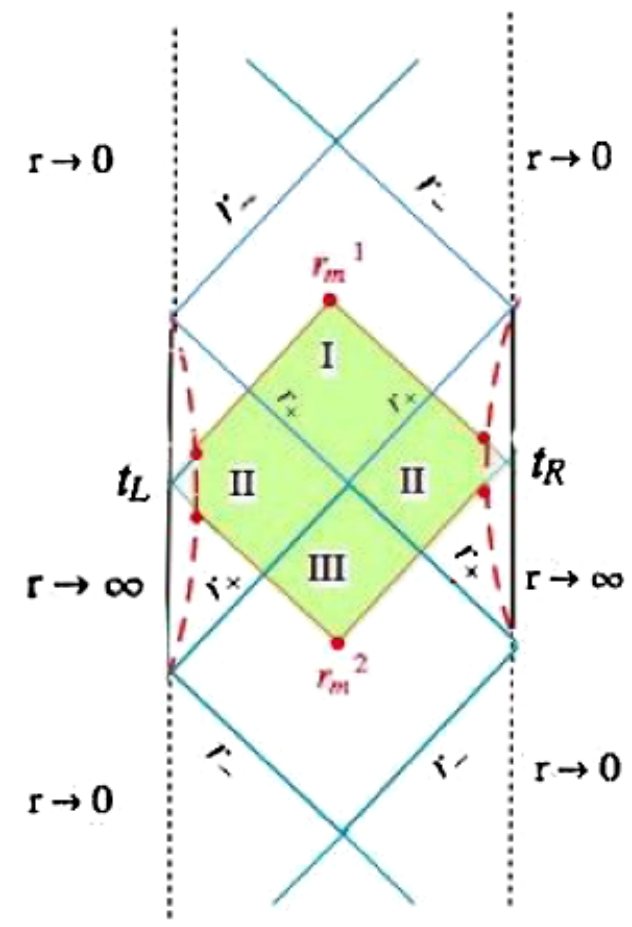

FIG. 7. Penrose diagram of the dyonic charged black hole. The Wheeler-DeWitt patch is shown in green.

and the chemical potentials are $\mu_{-}=Q / r_{-}, \mu_{+}=Q / r_{+}$, and $Q^{2}=q_{M}^{2}+q_{E}^{2}$.

The complexity growth rate for this solution at late times is [21]

$$
\frac{d S_{b k+b d}}{d t}=\left(M-\mu_{+} Q\right)-\left(M-\mu_{-} Q\right) .
$$

Note Fig. 7.

Now we find the full, time-dependent, holographic complexity. First, we divide the bulk action as

$$
\begin{aligned}
& I_{I}^{\text {bulk }}=\frac{\Omega_{2}}{8 \pi G_{N}} \int_{r_{+}}^{r_{\max }} d r\left[-\frac{2 r^{3}}{l^{2}}-\frac{2 r_{+} r_{-}}{r}\left(1+\frac{1}{l^{2}} \frac{r_{+}^{3}-r_{-}^{3}}{r_{+}-r_{-}}\right)\right]\left(\frac{t}{2}+r^{*}(0)-r^{*}(r)\right), \\
& I_{I I}^{\text {bulk }}=\frac{\Omega_{2}}{4 \pi G_{N}} \int_{r_{-}}^{r_{+}} d r\left[-\frac{2 r^{3}}{l^{2}}-\frac{2 r_{+} r_{-}}{r}\left(1+\frac{1}{l^{2}} \frac{r_{+}^{3}-r_{-}^{3}}{r_{+}-r_{-}}\right)\right]\left(r^{*}(0)-r^{*}(r)\right), \\
& I_{I I I}^{\text {bulk }}=\frac{\Omega_{2}}{8 \pi G_{N}} \int_{r_{+}}^{r_{m}} d r\left[-\frac{2 r^{3}}{l^{2}}-\frac{2 r_{+} r_{-}}{r}\left(1+\frac{1}{l^{2}} \frac{r_{+}^{3}-r_{-}^{3}}{r_{+}-r_{-}}\right)\right]\left(\frac{-t}{2}+r^{*}(0)-r^{*}(r)\right) .
\end{aligned}
$$

The sum of these terms is

$$
\begin{aligned}
I^{\text {bulk }}= & \frac{\Omega_{2}}{8 \pi G_{N}} \int_{r_{m}}^{r_{\max }} d r\left[-\frac{2 r^{3}}{l^{2}}-\frac{2 r_{+} r_{-}}{r}\left(1+\frac{1}{l^{2}} \frac{r_{+}^{3}-r_{-}^{3}}{r_{+}-r_{-}}\right)\right]\left(\frac{t}{2}-r^{*}(0)+r^{*}(r)\right), \\
& +\frac{\Omega_{2}}{4 \pi G_{N}} \int_{r_{-}}^{r_{\max }} d r\left[-\frac{2 r^{3}}{l^{2}}-\frac{2 r_{+} r_{-}}{r}\left(1+\frac{1}{l^{2}} \frac{r_{+}^{3}-r_{-}^{3}}{r_{+}-r_{-}}\right)\right]\left(r^{*}(0)-r^{*}(r)\right) .
\end{aligned}
$$


By choosing the normal vectors

$$
\begin{aligned}
& k_{1}^{a}=\alpha\left(\frac{1}{f(r)}\left(\partial_{t}\right)^{a}+\left(\partial_{r}\right)^{a}\right), \\
& k_{2}^{a}=\beta\left(-\frac{1}{f(r)}\left(\partial_{t}\right)^{a}+\left(\partial_{r}\right)^{a}\right)
\end{aligned}
$$

and the joint action

$$
I^{\text {joint }}=\frac{1}{8 \pi G_{N}} \int d^{d} x \sqrt{\gamma} \log \left|\frac{k_{1} \cdot k_{2}}{2}\right|,
$$

the contribution from this term at $r=r_{m}$ is

$$
I^{\text {joint }}=\frac{\Omega_{2}}{8 \pi G_{N}} r_{m}^{2} \log \left|\frac{\alpha \beta}{f\left(r_{m}\right)}\right| .
$$

Also, for the counterterm

$$
\frac{1}{8 \pi G_{N}} \int d \lambda d^{d} x \sqrt{\gamma} \log \frac{\Theta}{d}
$$

we find

$$
\Theta=\frac{1}{\sqrt{\gamma}} \frac{\partial \sqrt{\gamma}}{\partial \lambda}=\frac{2 \alpha}{r} .
$$

Here, $\lambda$ is the affine parameter for the null surface. For the null vector $k_{1}$ it is

$$
\frac{\partial r}{\partial \lambda}=\alpha
$$

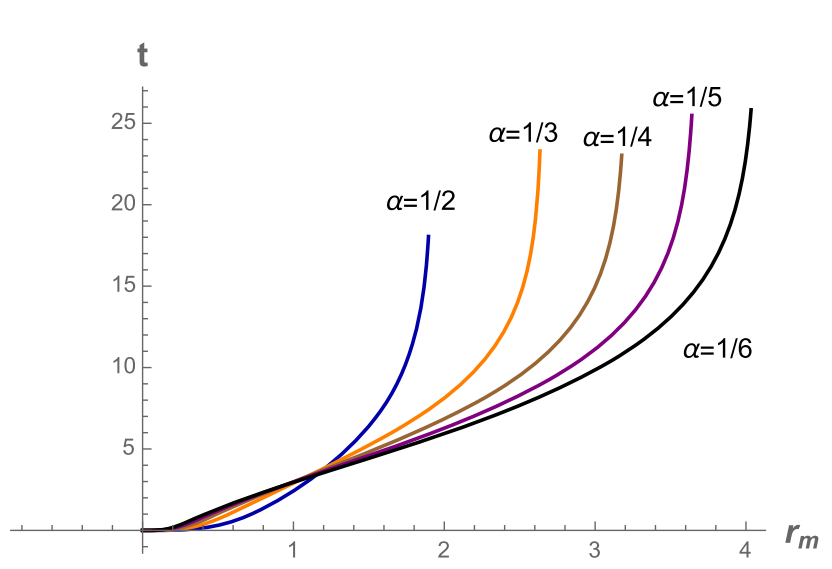

(a) time versus $r_{m}$
So, finally we get

$I^{c t}=\frac{\Omega_{2}}{8 \pi G_{N}} r_{m}^{2} \log |\alpha \beta|+\frac{\Omega_{2}}{8 \pi G_{N}}\left(r_{m}^{2}-2 r_{m}^{2} \log r_{m}\right)$,

where the first term can eliminate the ambiguity coming from the normalization factors of the null vectors. Summing all of these terms, taking the time derivative, removing some remaining constant terms, and using $\frac{d r_{m}}{d t}=-\frac{f\left(r_{m}\right)}{2}$, we finally get the relationship

$$
\begin{aligned}
\frac{d}{d t} \mathcal{C} \propto & -\frac{f\left(r_{m}\right) r_{m}^{3}}{16}\left(1+4 \log r_{m}\right)+f\left(r_{m}\right) r_{m} \log \left(\frac{f\left(r_{m}\right)}{r_{m}^{2}}\right) \\
& +\frac{r_{m}^{2} f^{\prime}\left(r_{m}\right)}{2} .
\end{aligned}
$$

The plots for the behaviors of the complexity of dyonic black holes are shown in Fig. 8(b), where $\alpha$ is just a constant defined as $\Lambda=m=q_{E}=q_{M}=\alpha$.

From these diagrams, one can see that the Lloyd bound at early times is violated, and at later times it is saturated from above, similar to other studies $[8,43,48,49]$.

In addition, we notice that by increasing the electric and magnetic charges $q_{E}$ and $q_{M}$, the maximum of $\frac{d C}{d t}$ increases and therefore the violation of the Lloyd bound at early times becomes stronger. Also, the limit of the Lloyd bound at late times increases as well. Note that this again could point to a relationship between quantum fluctuations (like the Schwinger mechanism) and the complexity growth rate, as we have conjectured before. Of course, to study this relation further one could study the Schwinger effect for other charged black holes with various gauge field setups. For instance, the electric and magnetic fields might have different directions with respect to each other, and then one could study the complexity growth rate for those various angle setups, and therefore further quantify this connection.

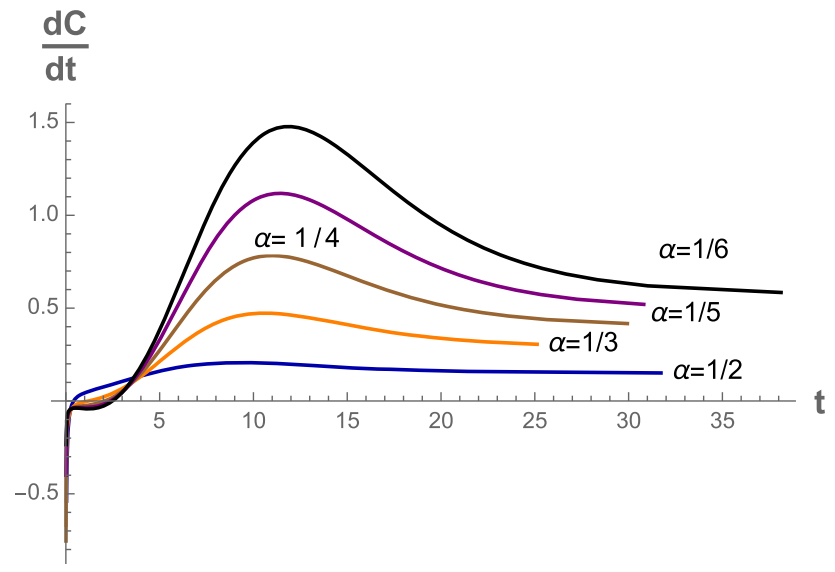

(b) complexity growth rate versus time

FIG. 8. The full time behaviors of the complexity growth rate of dyonic black holes. 


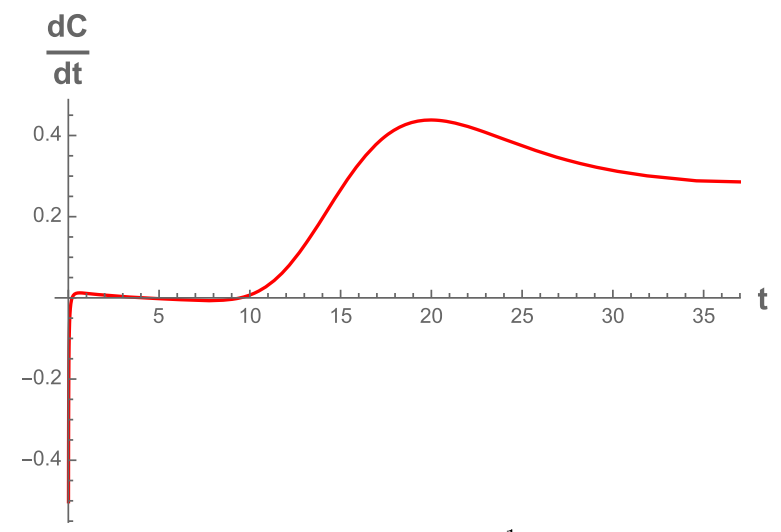

(a) $q_{E}=q_{M}=\frac{1}{4}$

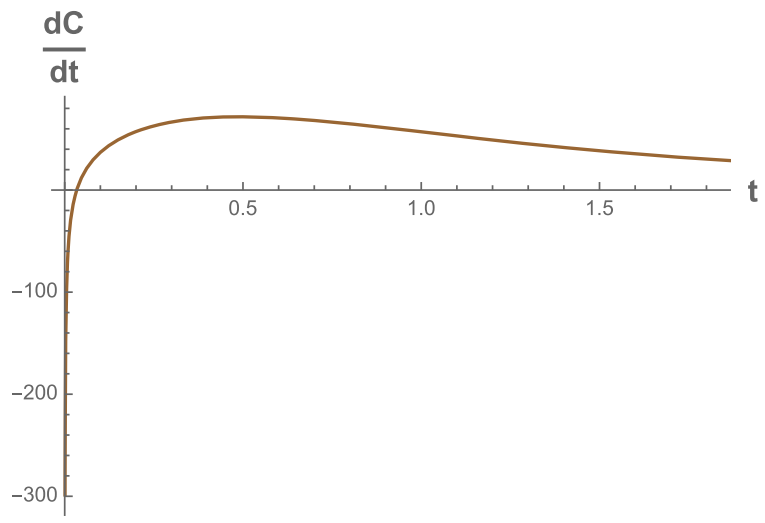

(c) $q_{E}=q_{M}=10$

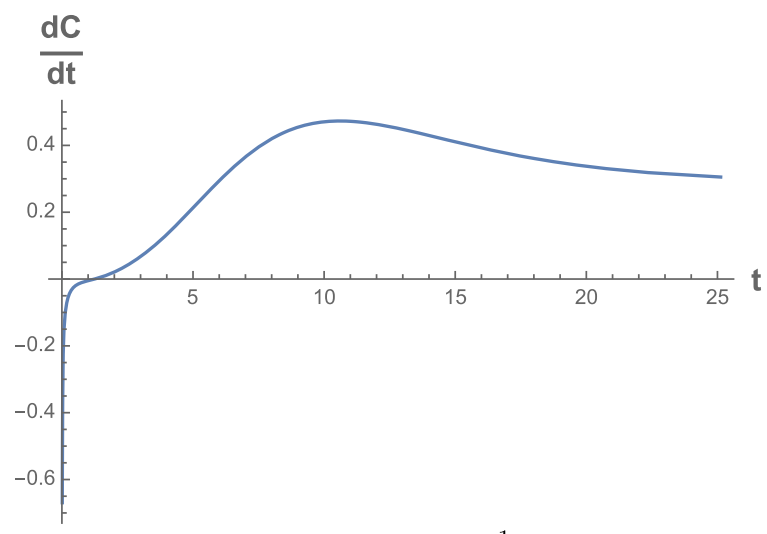

(b) $q_{E}=q_{M}=\frac{1}{3}$

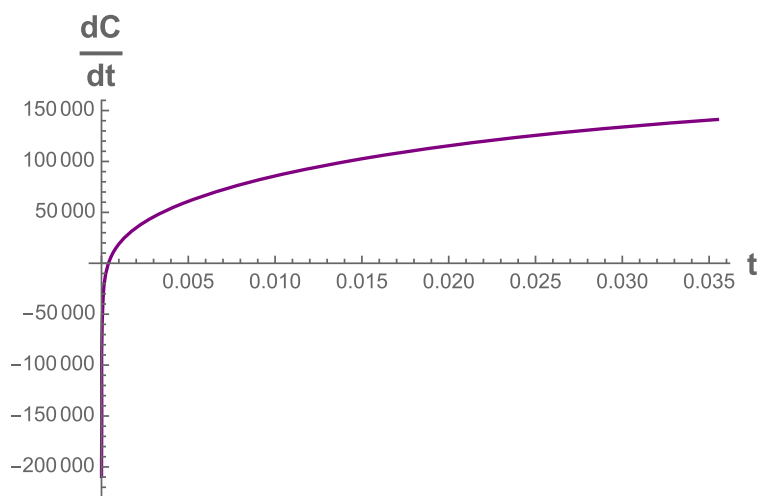

(d) $q_{E}=q_{M}=200$

FIG. 9. The change of the behavior of the complexity growth rate by changing the charges of dyonic black holes.

Additionally, from these diagrams we see that keeping the charges constant while increasing $m$ or $\Lambda$ decreases the maxima in the plots of $\frac{d C}{d t}$ versus $t$ as well as the final latetime limit, which is the Lloyd bound. It would also decrease the time which is needed to reach to that specific Lloyd bound.

By keeping $m$ and $\Lambda$ constant and changing $q_{E}$ and $q_{M}$, one can observe different phases which are shown in the plots of $\frac{d C}{d t}$ versus time in Fig. 9.

We see that for very small charges, the complexity growth rates at the beginning are very close to zero and only fluctuate a little bit at early times, suddenly increase and reach their maximum, and finally at late times approach the corresponding Lloyd bound [Fig. 9(a)].

Increasing the charges would smooth out the fluctuations at the beginning and make the complexity growth rate reach its maximum (and thus its Lloyd bound) much faster, which can be seen from the time axes in Figs. 9(b) and 9(c).

For very large charges the complexity growth rate diverges. This means that the dyonic black hole is unstable for this range of charge; see Fig. 9(d). So, in addition to the various phase transitions, the complexity growth rate could capture the instabilities in the solutions of black holes.
One could also consider some additional counterterms for these dyonic black holes (similar to Ref. [43]) and their effects. This might solve the peculiar behavior at the beginning of our diagrams, or it might even solve the issue of the violation of the Lloyd bound at early times.

Note that as the dyonic black holes are the dual holographic model for van der Waals fluids [47,50,51], we expect that the behavior of the complexity growth rate for these systems would be similar to the diagrams in Fig. 8(b) and this could probably be tested in the lab.

\section{B. Complexity in the AdS soliton background}

In this section we study the AdS soliton solution, which is a confining geometry but still very similar to the conformal AdS case, as it can be transformed to it by a double Wick rotation.

The AdS soliton background is

$$
\begin{aligned}
d s^{2}= & \frac{r^{2}}{\ell^{2}}\left[-d t^{2}+\left(1-\frac{r_{+}^{d}}{r^{d}}\right) d \chi^{2}+d \vec{x}^{2}\right] \\
& +\left(1-\frac{r_{+}^{d}}{r^{d}}\right)^{-1} \frac{\ell^{2}}{r^{2}} d r^{2},
\end{aligned}
$$


where $\chi$ is the circle with the smallest period that has antiperiodic boundary conditions. The periodicity of $\chi, \Delta \chi$, and the parameter $r_{+}$are related by $\Delta \chi=\frac{4 \pi \ell^{2}}{d r_{+}}$, which comes from the smoothness at $r=r_{+}$. Note that as the boundary here is $d$ dimensional, there are $d-2$ coordinates $\vec{x}$ and the volume of these transverse $d-2 x^{i}$, s would be $V_{x}$.

This solution has the following negative boundary energy [31]:

$$
E=-\frac{r_{+}^{d} \Delta \chi V_{x}}{\ell^{d+1}}=-\frac{V_{x} \ell^{d-1}(4 \pi)^{d}}{d^{d} \Delta \chi^{d-1}} .
$$

This ground state energy is the result of the variance between the Casimir energies of bosons and fermions, which exists due to the antiperiodic boundary conditions for the fermions. Increasing the antiperiodicity would increase this negative Casimir energy, and thus the complexity.

Using the CV conjecture, the complexity in the AdS soliton case is [31]

$$
\mathcal{C}_{V}=\frac{8 V_{x} \Delta \chi}{\pi} \frac{r_{\max }^{d-1}-r_{+}^{d-1}}{\ell^{d-1}}
$$

where $r=r_{\max }$ is a UV cutoff.

We note that increasing the periodicity $\Delta \chi$ and volume $V_{x}$ linearly increases the complexity. This result could be confirmed by calculating the complexity using the CA conjecture as well [31].

Note that for the case of pure AdS the complexity is $\mathcal{C}_{V}=\frac{8 e^{d-1} V_{x}}{\pi \epsilon^{d-1}}$, where $\epsilon$ is the cutoff in the radial direction. So from the $\mathrm{CV}$ conjecture one can find that the difference between these two complexities is negative and is in fact the second term of Eq. (3.15). So, from Eq. (3.15) we see that the complexity of the AdS soliton case is smaller than that of the AdS case. This can be explained by the fact that, relative to the AdS soliton case, the pure AdS case is the excited state and therefore (as its energy is higher) the complexity of the AdS background would also be higher than in the AdS soliton background.

However, using the CA conjecture the authors of Ref. [31] found a different result for the relation between the complexity and $r_{+}$. Using the CA conjecture, they calculated the full IR/UV behavior of the complexity growth rate and found that as the IR scale increases, the action first increases until reaching a maximum, and then decreases to zero at the UV cutoff. They justified this result (that the complexity of the AdS soliton is higher that of the AdS case) by calculating the complexity using Nielsen approach [52] and from field theory calculations for fermions on a rectangular lattice. However, during that calculation they used the Manhattan metric and made many

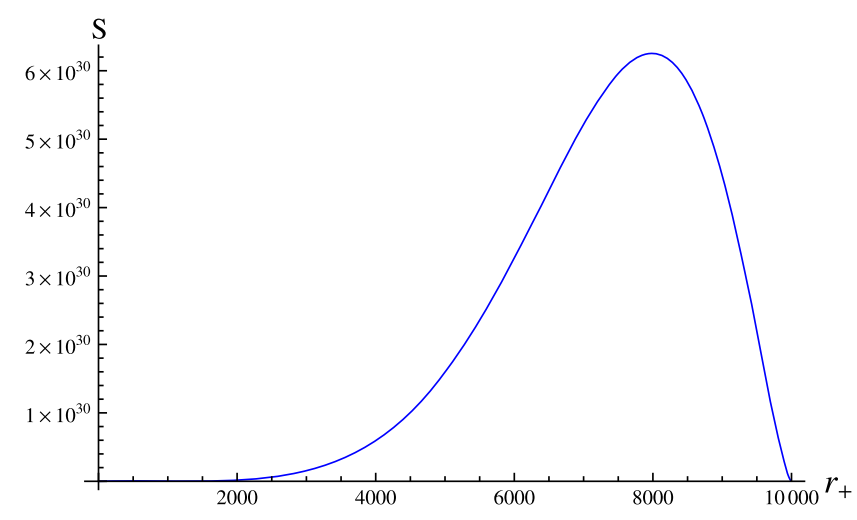

FIG. 10. $\quad S$ versus $r_{+}$for $d=4, r_{\max }=10000$, and $l=1$.

approximations, and thus using other metrics or assumptions could actually change the final result.

For instance, by considering gates with penalty factors that could take into account both the entanglement between such gates and nonlocality, one could again calculate the complexity. Doing this might change the result of Ref. [31] from field theory and lattice calculations, so then their calculation of the complexity from the $\mathrm{CV}$ conjecture would match the result from field theory.

We reproduce their plot for the whole action versus the radial distance for the case of the AdS soliton in Fig. 10, which actually comes from Eq. (38) of Ref. [31]. Note that there is no time dependence here, as time is constant and we plot the complexity rather than its growth rate. The complexity growth rate for the AdS soliton could be studied in future works.

We could examine which one of their results would be more compatible with other observations regarding the relationship between complexity and other physical quantities such as changes in the potential. Comparing the behavior of the complexity, potentials, and phase diagrams of Schwinger pair creation in the AdS and AdS soliton backgrounds (Fig. 11) could give some hint about the correct answer for the complexity.

In Ref. [53] it was shown that there is a connection between AdS bubbles (AdS solitons) and closed string tachyon condensations, and it was also found that the degrees of freedom and therefore the entanglement entropy would decrease under this tachyon condensation (which is a second-order phase transition). In addition, the energy density would decrease in this process [54,55]. So, because both the energy and entanglement entropy of the AdS soliton are lower than in the AdS case, and because the AdS soliton is more stable than the AdS background, one could propose that the complexity of the AdS soliton should be lower than the AdS case, and therefore that the calculation using the CV conjecture in Ref. [31] is the correct answer. One could repeat the calculations for the "twisted" AdS bubble which is a better gravity dual of the corresponding Yang-Mills theory on $S^{1} \times R^{3}$ and compare the results. 


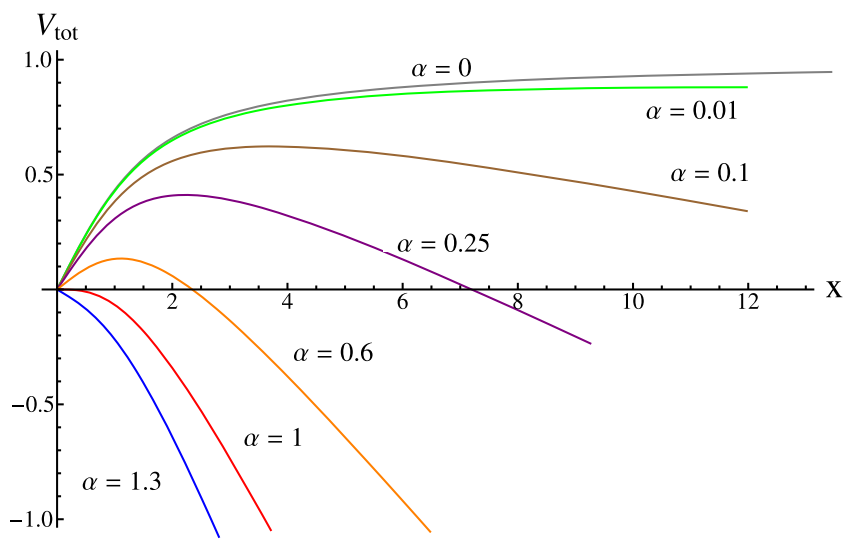

(a) $\operatorname{AdS}$

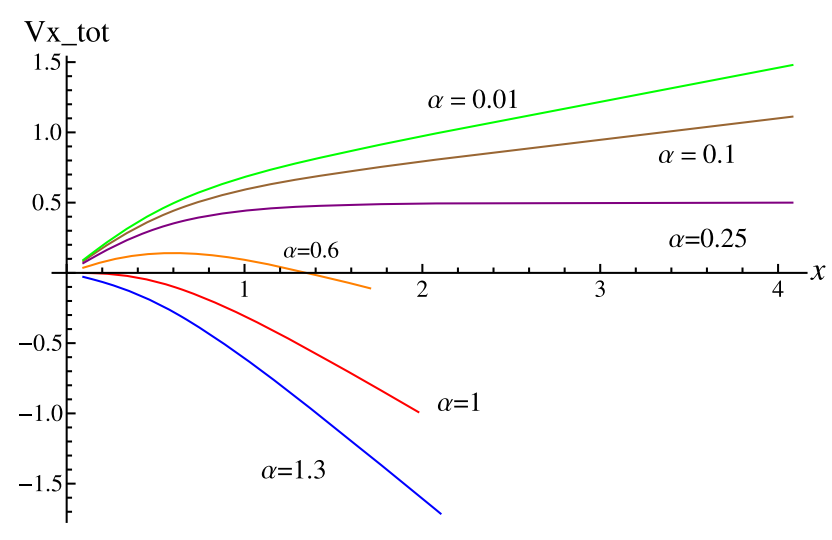

(b) AdS soliton

FIG. 11. Comparing the Schwinger phase diagrams of the AdS and AdS soliton cases.

Moreover, recently in Ref. [56] it was found that among different holographic (CV and $\mathrm{CA}$ ) and field theory methods [Fubini-Study metric (FS) and Finsler geometry (FG)] for calculating complexity, the holographic CV conjecture and field-theoretic FG method are actually correlated. So if one actually repeated the calculation of Ref. [31] for the AdS soliton using FG, a result more correlated with CV could be derived. We hope to come back to this problem in future works.

To get further information, one could compare the phase diagrams of the Schwinger effect in both the AdS and AdS soliton backgrounds while an electric field is present.

At each value of $\alpha$ (which here is the dimensionless quantity for the strength of the electric field, i.e., $\alpha=\frac{E}{E_{c}}$ ), the potential of the AdS soliton would be higher, pointing to smaller fluctuations and therefore smaller complexity. There are also three phases in the AdS soliton background, while there are two phases for the AdS case. These richer possibilities for the phases could also be seen from the behavior of complexity.

The quasinormal modes in the AdS soliton case were plotted in Ref. [57]. We note that for higher radial distances these modes are exponentially damped, which explains the decrease of complexity.

This connection could be examined further in future works. For instance, one could turn on a magnetic field in addition to the electric field and change the angle between the directions of the two, which could significantly change the Schwinger pair creation rate. As a universal result, Ref. [58] found that when the electric field is parallel to the magnetic field the pair creation rate is higher relative to the setup where they are perpendicular. The complexity growth rates in these two setups would also be different. The results could be compared with the pair creation rates to find more evidence for the connection between quantum fluctuations, boundary periodicity, and complexity.

\section{COMPLEXITY AND PHASE TRANSITIONS IN QCD MODELS}

To further study the relationship between potentials, quantum fluctuations, phase transitions, and complexity growth, we study the Gubser model of QCD [59-63], which is the main part of this work.

By tuning the parameters of the potential, in this model several kinds of phase transitions can be displayed. Based on the parameters of the dilaton potential, it can generate three types of phase transitions: crossover, first order, and second order. We study the full time behavior of complexity growth rates around these phase transitions and their correlations with other thermodynamical quantities.

In Ref. [36], by using the CA conjecture, the late-time behavior of the complexity of this holographic QCD model was studied. There, it was shown that the complexity growth rate can also be used as a parameter to detect the phase transitions. This is because the behavior of the entropy and complexity growth rate versus temperature would be the same for each type of phase transition. We then study the connection between the behaviors of the complexity growth rate, speed of sound, entropy, and potential for four different models of confining potentials.

The action of this model is

$$
\begin{aligned}
S= & \frac{1}{16 \pi G_{5}} \int d^{5} x \sqrt{-g}\left[R-\frac{1}{2}(\partial \phi)^{2}-V(\phi)\right] \\
& +\frac{1}{8 \pi G_{5}} \int_{\partial \mathcal{M}} K,
\end{aligned}
$$


where $K$ is the trace of the extrinsic curvature. For simplicity, we also take $16 \pi G_{5}=1$ and $\hbar=1$.

In this model the five-dimensional gravity is coupled to a single scalar field. This setup then contains the minimum freedom needed to match the equation of states and also reproduce the desired phase diagrams of QCD.

The general ansatz for the dilaton potential is

$V(\phi)=-12\left(1+a \phi^{2}\right)^{1 / 4} \cosh (\gamma \phi)+b_{2} \phi^{2}+b_{4} \phi^{4}+b_{6} \phi^{6}$,

where $\left(a, \gamma, b_{2}, b_{4}, b_{6}\right)$ are the parameters shown below.

\begin{tabular}{lccccccc} 
Potential & $a$ & \multicolumn{1}{c}{$\gamma$} & $b_{2}$ & $b_{4}$ & \multicolumn{1}{c}{$b_{6}$} & $\Delta$ & $T_{c}$ \\
\hline$V_{\mathrm{QCD}}$ & 0 & 0.606 & 1.4 & -0.1 & 0.0034 & 3.55 & 0.181033 \\
$V_{\text {2nd }}$ & 0 & $1 / \sqrt{2}$ & 1.958 & 0 & 0 & 3.38 & 0.243901 \\
$V_{1 \text { st }}$ & 0 & $\sqrt{7 / 12}$ & 2.5 & 0 & 0 & 3.41 & 0.156841 \\
$V_{\text {IHQCD }}$ & 1 & $\sqrt{2 / 3}$ & 6.25 & 0 & 0 & 3.58 & 0.295847
\end{tabular}

To compare the behavior of the potential with regard to the different parameters, which would then lead to various phase transitions, we plot $V(\phi)$ and $\frac{\partial_{\phi} V}{V}$ versus $\phi$ in Fig. 12. The parameters in this potential $[59,60,64]$ have been chosen in such a way that the plots of $c_{s}^{2}$ versus $T / T_{c}$ would match the phenomenological results of a hadron gas and the lattice models. We discuss the properties of each model later in this section.

First, we consider the following ansatz:

$$
d s^{2}=e^{2 A}\left(-h d t^{2}+d \vec{x}^{2}\right)+\frac{e^{2 B}}{h} d r^{2}, \quad \phi=r,
$$

where $A, B$, and $h$ are only functions of $r$ (or $\phi$ ). Note that the asymptotic boundary is at $r \rightarrow 0$ and the singularity is where $r \rightarrow \infty$.

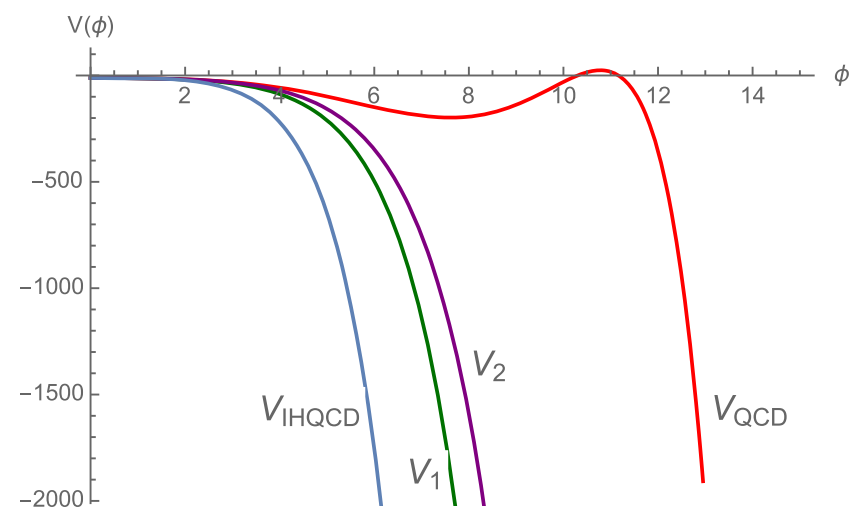

(a) $V(\phi)$ vs. $\phi$.
The equations of motion are

$$
\begin{aligned}
A^{\prime \prime}-A^{\prime} B^{\prime}+\frac{1}{6} & =0, \\
h^{\prime \prime}+\left(4 A^{\prime}-B^{\prime}\right) h^{\prime} & =0, \\
6 A^{\prime} h^{\prime}+h\left(24 A^{\prime 2}-1\right)+2 e^{2 B} V & =0, \\
4 A^{\prime}-B^{\prime}+\frac{h^{\prime}}{h}-\frac{e^{2 B}}{h} V^{\prime} & =0,
\end{aligned}
$$

and the horizon is where $h\left(\phi_{H}\right)=0$.

In Ref. [65], using the method of Refs. [59,60], the field equations were solved as

$$
\begin{aligned}
& A(\phi)=A_{H}+\int_{\phi_{H}}^{\phi} d \tilde{\phi} G(\tilde{\phi}), \\
& B(\phi)=B_{H}+\ln \left(\frac{G(\phi)}{G\left(\phi_{H}\right)}\right)+\int_{\phi_{H}}^{\phi} \frac{d \tilde{\phi}}{6 G(\tilde{\phi})}, \\
& h(\phi)=h_{H}+h_{1} \int_{\phi_{H}}^{\phi} d \tilde{\phi} e^{-4 A(\tilde{\phi})+B(\tilde{\phi}),}
\end{aligned}
$$

where $G(\phi) \equiv A^{\prime}(\phi)$. Then, using the initial and boundary conditions, the constants were found to be

$$
\begin{aligned}
A_{H} & =\frac{\ln \phi_{H}}{\Delta-4}+\int_{0}^{\phi_{H}} d \phi\left[G(\phi)-\frac{1}{(\Delta-4) \phi}\right], \\
B_{H} & =\ln \left(-\frac{4 V\left(\phi_{H}\right)}{V(0) V^{\prime}\left(\phi_{H}\right)}\right)+\int_{0}^{\phi_{H}} \frac{d \phi}{6 G(\phi)}, \\
h_{H} & =0 \\
h_{1} & =\frac{1}{\int_{\phi_{H}}^{0} d \phi e^{-4 A(\phi)+B(\phi)}} .
\end{aligned}
$$

To find the solution of $G(\phi)$, one actually needs to solve the equation

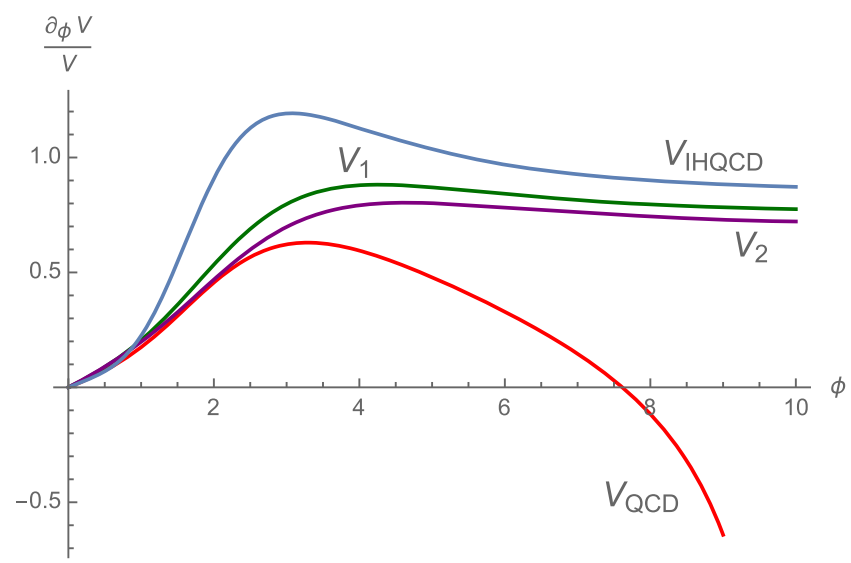

(b) $\frac{\partial_{\phi} V}{V}$ vs. $\phi$.

FIG. 12. QCD potentials and their derivative versus $\phi$. 


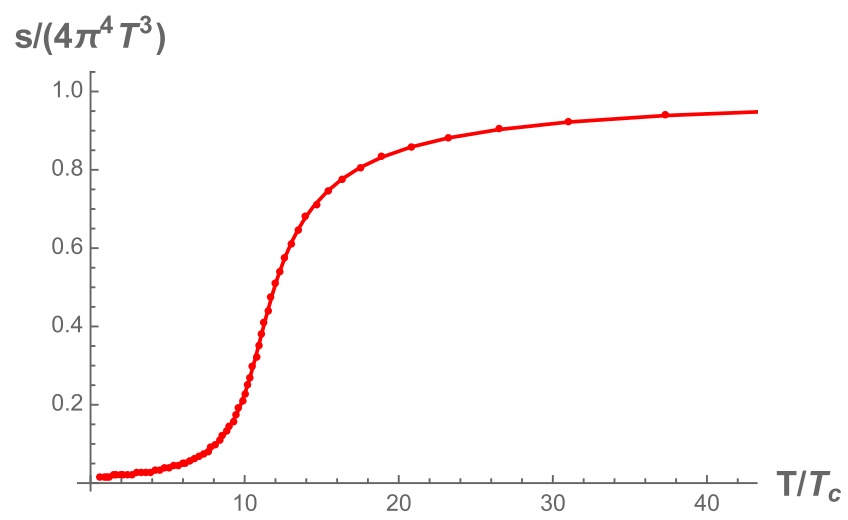

(a) $V_{Q C D}$

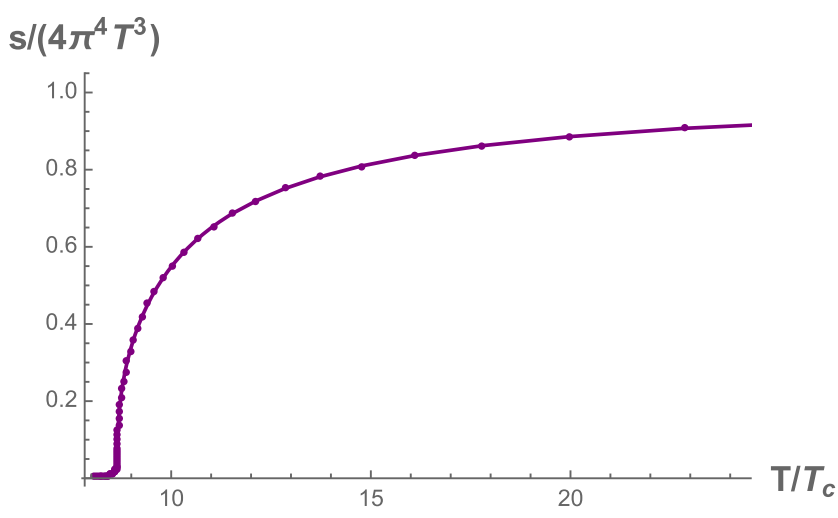

(c) $V_{2}$

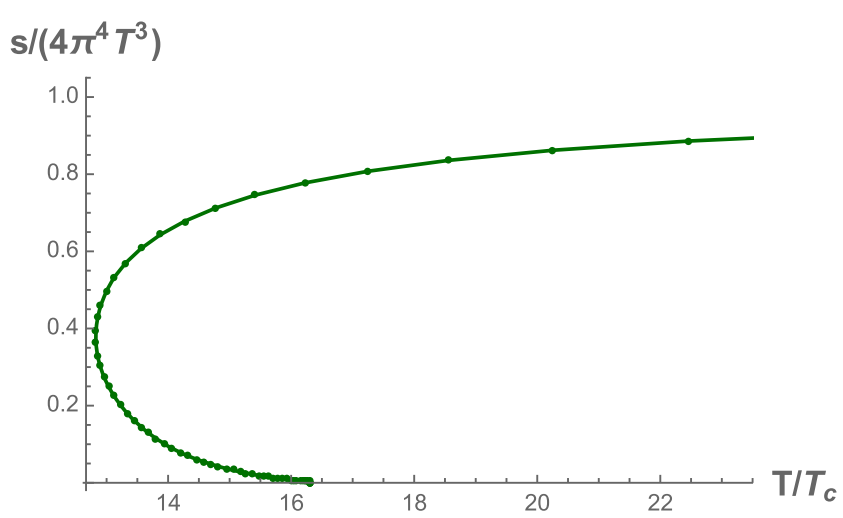

(b) $V_{1}$

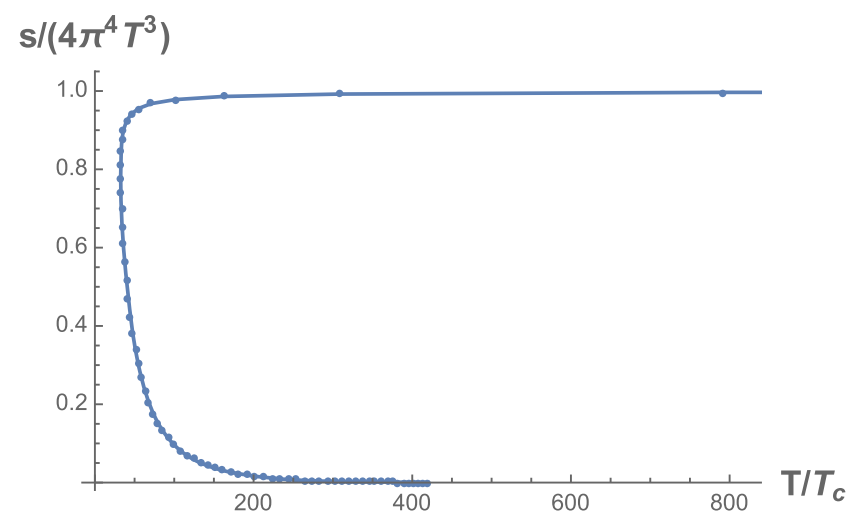

(d) $V_{I H Q C D}$

FIG. 13. Phase transition of entropy versus temperature for four nonconformal cases.

$\frac{G^{\prime}}{G+V / 3 V^{\prime}}=\frac{d}{d \phi} \ln \left(\frac{G^{\prime}}{G}+\frac{1}{6 G}-4 G-\frac{G^{\prime}}{G+V / 3 V^{\prime}}\right)$,

from which one can find the series expansion of $G(\phi)$ near the horizon $\phi=\phi_{H}$ as

$$
\begin{aligned}
G(\phi)= & -\frac{V(\phi)}{3 V^{\prime}(\phi)}+\frac{1}{6}\left(\frac{V\left(\phi_{H}\right) V^{\prime \prime}\left(\phi_{H}\right)}{V^{\prime}\left(\phi_{H}\right)^{2}}-1\right)\left(\phi-\phi_{H}\right) \\
& +\mathcal{O}\left(\phi-\phi_{H}\right)^{2} .
\end{aligned}
$$

This result can be used as the boundary condition to solve $G(\phi)$.

Assuming $16 \pi G_{5}=1$ and $\hbar=1$, the temperature, entropy, and speed of sound $c_{s}$ are written as

$$
\begin{aligned}
T & =\frac{e^{A_{H}-B_{H}}}{4 \pi}\left|h^{\prime}\left(\phi_{H}\right)\right|=\frac{e^{A_{H}+B_{H}}}{4 \pi}\left|V^{\prime}\left(\phi_{H}\right)\right|, \quad s=4 \pi e^{3 A_{H}}, \\
c_{s}^{2} & =\frac{d \log T}{d \log s} .
\end{aligned}
$$

We can solve for these parameters numerically, and the plots of the entropy and speed of sound versus temperature for the four potential models are shown in Figs. 13 and 14. Note that in all of these models $s / T^{3}$ is actually proportional to the number of degrees of freedom [65]. Therefore, one would expect that the behavior of the complexity growth rate would be similar to the entropy.

For the late-time interval $[t, t+\delta t]$, the bulk term of the on-shell action inside the horizon is

$$
\begin{aligned}
S_{\text {bulk }} & =\int d^{5} x \frac{2}{3 \pi G_{N}} e^{4 A+B} V(r) \\
& =\frac{2 V_{3}}{3 \pi G_{N}} \int_{t}^{t+\delta t} d t \int_{r_{H}}^{\infty} e^{4 A+B} V(r) d r,
\end{aligned}
$$

and the Gibbons-Hawking-York (GHY) term is

$$
S_{\mathrm{GHY}}=\left.\delta t V_{3}\left[e^{-4 A-B} \partial_{r}\left(e^{8 A} h\right)\right]\right|_{r_{H}} ^{\infty},
$$

where $V_{3} \equiv \int d^{3} \vec{x}$ is the volume of the boundary field system.

So the growth rate of the holographic complexity density $c \equiv \mathcal{C} / V_{3}$ at late time is 


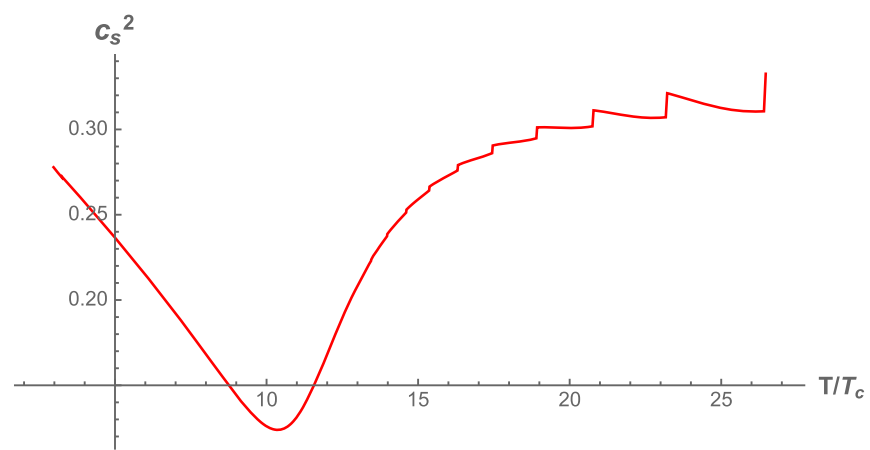

(a) $V_{Q C D}$

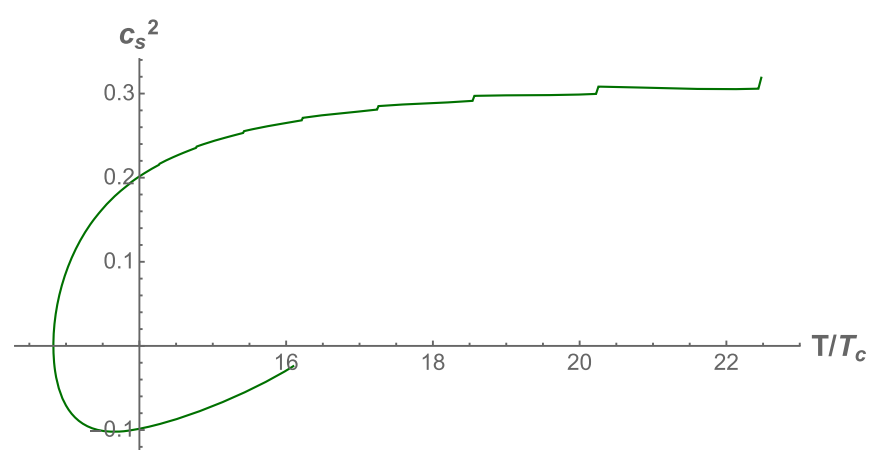

(b) $V_{1}$

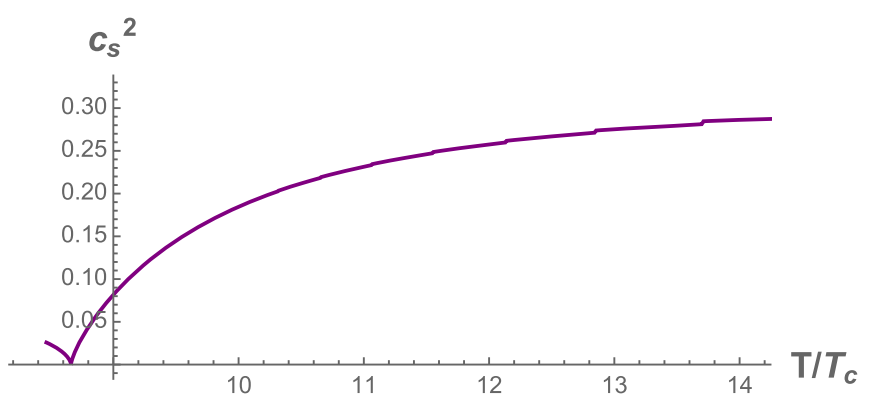

(c) $V_{2}$

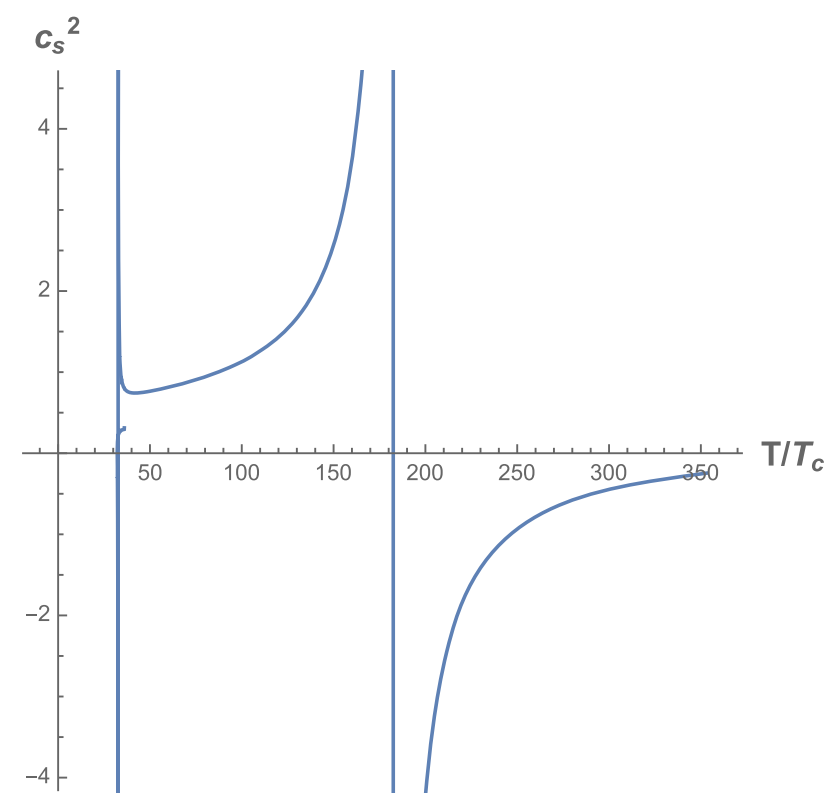

(d) $V_{I H Q C D}$

FIG. 14. Speed of sound versus $T / T_{c}$ for four nonconformal cases.

$\frac{d c}{d t}=\frac{2}{3 \pi G_{N}} \int_{r_{H}}^{\infty} e^{4 A+B} V(r) d r+\left.\frac{1}{\pi}\left[e^{-4 A-B} \partial_{r}\left(e^{8 A} h\right)\right]\right|_{r_{H}} ^{\infty}$.

We plot the behavior of the late-time complexity during each phase transition are presented in Fig. 15. As one would expect and as found in Ref. [36], they are very similar to the diagrams of the entropy for each potential, demonstrating that complexity could also act as a good probe of confinement and phase transitions.

Interestingly, the jump that we see in the complexity for all of these cases during the phase transition is close to the topological jump of $\Delta \mathcal{C}=2 \pi$ found in Refs. [12,26]. So it is worth noting that the results coming from the "complexity = action" conjecture are fairly consistent with the results of Ref. [12] which came from the "complexity = volume" and the "subregion complexity" conjectures $[4,5,66,67]$.
Comparing these diagrams with those for the speed of sound [68] would be interesting too. Note that for high temperatures where the first three models become conformal, the speed of sound becomes constant and close to 0.3 , and the complexity growth rate would become constant as well. For the low-temperature case, note that when the slope of the $c_{s}^{2}$ versus $T / T_{c}$ diagram is negative, the slope of the $c_{s}^{2}$ versus $T / T_{c}$ diagram would be positive, and vice versa. This could be qualitatively explained by the fact that when the speed of sound grows in a region, information can propagate easier; thus, it would be easier to go from one state to another, and therefore the complexity growth rate would decrease.

Now we study the full time behavior of the complexity growth rate in this model. Similar to Refs. $[8,69]$, the time evolution of complexity can be found by adding the null boundary and joint terms to the action and GHY terms: 


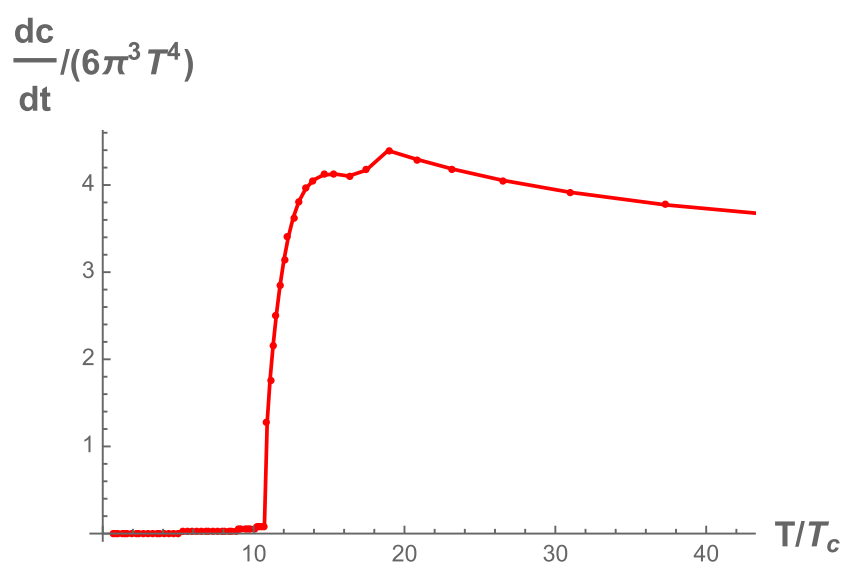

(a) $V_{Q C D}$

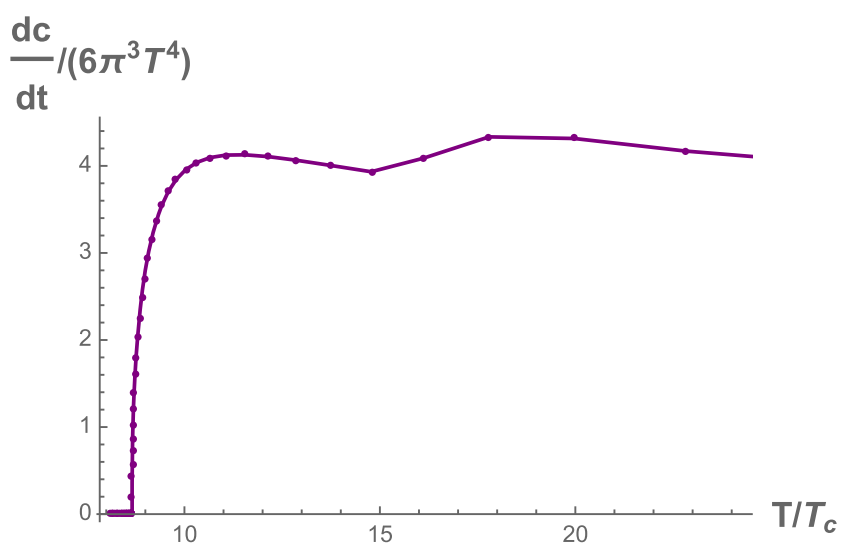

(c) $V_{2}$ $\frac{\mathrm{dc}}{\mathrm{dt}} /\left(6 \pi^{3} T^{4}\right)$

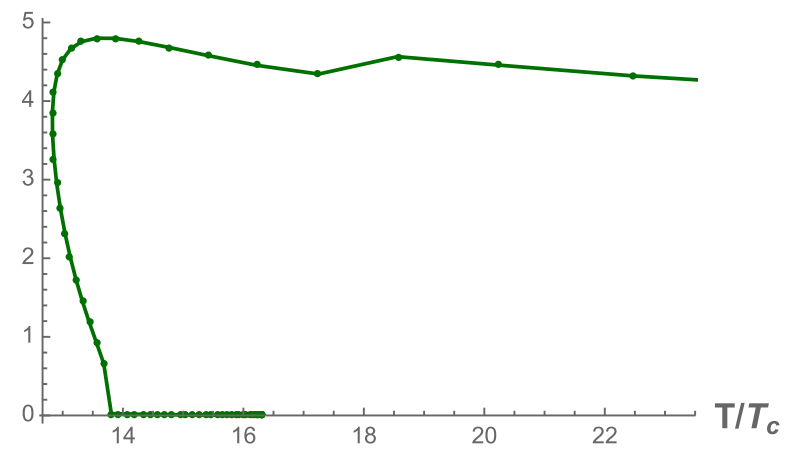

(b) $V_{1}$

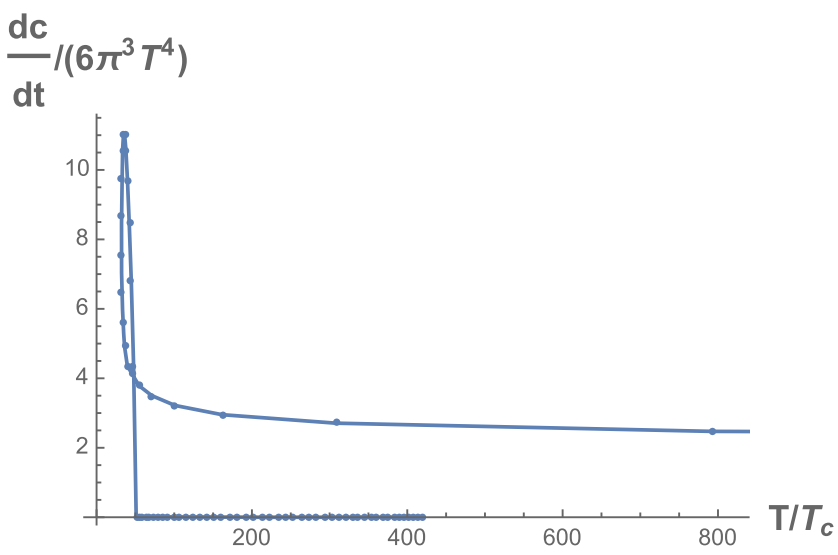

(d) $V_{I H Q C D}$

FIG. 15. Phase transition of the complexity growth rate versus temperature for four nonconformal cases.

$$
\begin{aligned}
I= & \frac{1}{16 \pi G_{N}} \int_{\mathcal{M}} d^{5} x \sqrt{-g}\left[R-\frac{1}{2}(\partial \phi)^{2}-V(\phi)\right] \\
& +\frac{1}{8 \pi G_{N}} \int_{\mathcal{B}} d^{4} x \sqrt{|h|} K+\frac{1}{8 \pi G_{N}} \int_{\Sigma} d^{3} x \sqrt{\sigma} \eta \\
& -\frac{1}{8 \pi G_{N}} \int_{\mathcal{B}^{\prime}} d \lambda d^{3} \theta \sqrt{\gamma} \kappa+\frac{1}{8 \pi G_{N}} \int_{\Sigma^{\prime}} d^{3} x \sqrt{\sigma} a .
\end{aligned}
$$

The affine parametrization could be chosen in such a way that $\kappa=0$ so as to make the contribution of the null boundary vanish.

Now, assuming $G_{N}=1$ and $t_{L}=t_{R}=\frac{t}{2}$, we can divide the evolution of the black hole into two stages: the time before the critical time $t_{c}$, and after it. Before the critical time, the past null boundary intersects the past singularity and there is a GHY boundary term. After the critical time, however, the two null boundaries from the left and right conformal field theories intersect, and therefore there would be a contribution from the null joint term instead of a GHY term.
The critical time $t_{c}$ can be found by using

$$
\begin{aligned}
\frac{t_{c}}{2}-r^{*}(\infty) & =t-r^{*}(0), \\
-\frac{t_{c}}{2}+r^{*}(\infty) & =t+r^{*}(0),
\end{aligned}
$$

so that

$$
t_{c}=2\left(r^{*}(\infty)-r^{*}(0)\right) .
$$

From Fig. 16, for a state at $r_{R}=t_{L}=\frac{t}{2}>\frac{t_{c}}{2}$, one gets

$$
\begin{aligned}
S_{\text {bulk }_{I}} & =\frac{2 V_{3}}{3 \pi G_{N}} \int_{r_{H}}^{r_{\max }} d r e^{4 A+B} V(r)\left(\frac{t}{2}+r^{*}(0)-r^{*}(r)\right), \\
S_{\text {bulk }_{I I}} & =\frac{4 V_{3}}{3 \pi G_{N}} \int_{\delta}^{r_{H}} d r e^{4 A+B} V(r)\left(r^{*}(0)-r^{*}(r)\right), \\
S_{\text {bulk }_{I I I}} & =\frac{2 V_{3}}{3 \pi G_{N}} \int_{r_{H}}^{r_{m}} d r e^{4 A+B} V(r)\left(-\frac{t}{2}+r^{*}(0)-r^{*}(r)\right),
\end{aligned}
$$




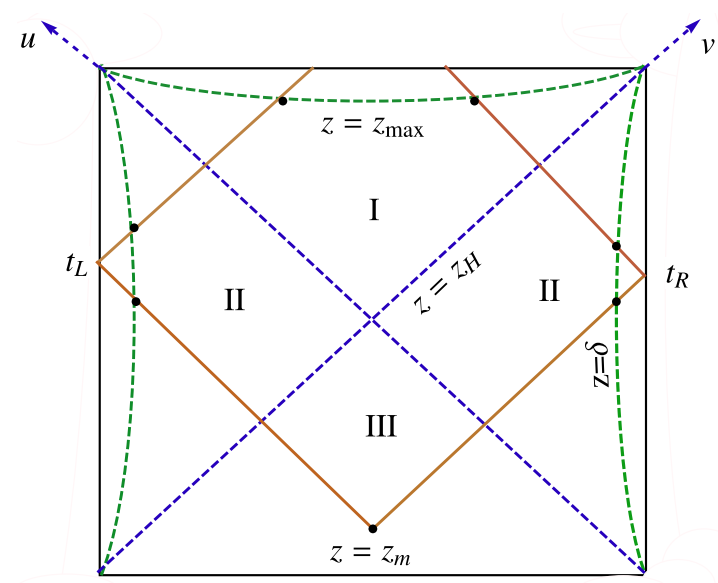

FIG. 16. Penrose diagram for the black brane ansatz and the null boundaries.

so that

$$
\begin{aligned}
S_{\text {bulk }}= & \frac{4 V_{3}}{3 \pi G_{N}} \int_{\delta}^{r_{\max }} d r e^{4 A+B} V(r)\left(r^{*}(0)-r^{*}(z)\right) \\
& +\frac{2 V_{3}}{3 \pi G_{N}} \int_{r_{m}}^{r_{\max }} d r e^{4 A+B} V(r)\left(\frac{t}{2}-r^{*}(0)+r^{*}(r)\right) .
\end{aligned}
$$

Note that $r^{*}(r)=\int \frac{e^{B-A}}{h} d r$. Also, the GHY boundary term at $r=r_{\max }$ is

$$
\begin{aligned}
S_{\text {bound }}= & \frac{V_{3}}{8 \pi G_{N}}\left(\frac{t}{2}+r^{*}(0)-r^{*}\left(r_{\max }\right)\right) \\
& \times\left(e^{-4 A-B} \partial_{r}\left(e^{8 A} h\right)\right)_{r=r_{\max }} .
\end{aligned}
$$

Now, to consider the contribution of joint terms (which occurs at $r=r_{m}$ ), we first take two normal vectors of the null boundaries,

$$
\begin{aligned}
& k_{1}^{a}=\alpha\left(\frac{e^{-2 A}}{h}\left(\partial_{t}\right)^{a}+e^{-A-B}\left(\partial_{r}\right)^{a}\right), \\
& k_{2}^{a}=\beta\left(-\frac{e^{-2 A}}{h}\left(\partial_{t}\right)^{a}+e^{-A-B}\left(\partial_{r}\right)^{a}\right) .
\end{aligned}
$$

Then, the joint action

$$
S_{\text {joint }}=\frac{1}{8 \pi G_{N}} \int d^{d} x \sqrt{\gamma} \log \left|\frac{k_{1} \cdot k_{2}}{2}\right|
$$

would be

$$
\begin{aligned}
S_{\text {joint }}= & \frac{V_{3}}{8 \pi G_{N}} e^{3 A\left(r_{m}\right)}\left(\log \left|e^{2 A\left(r_{m}\right)}\right|-\log \left|h\left(r_{m}\right)\right|\right) \\
& +\frac{V_{3}}{8 \pi G_{N}} e^{3 A\left(r_{m}\right)} \log \alpha \beta .
\end{aligned}
$$

In the above action, $\gamma$ is the determinant of the induced metric on the joint point and $\alpha$ and $\beta$ are two constants that appear because of the ambiguity in the normalization of the null boundaries. To remove the ambiguity, one can add the following counterterm:

$$
\frac{1}{8 \pi G_{N}} \int d \lambda d^{d} x \sqrt{\gamma} \log \frac{\Theta}{d}
$$

where

$$
\Theta=\frac{1}{\sqrt{\gamma}} \frac{\partial \sqrt{\gamma}}{\partial \lambda} .
$$

Here, $\lambda$ is the affine parameter for the null surface. For the null vector $k_{1}$ it would be

$$
\frac{\partial r}{\partial \lambda}=\alpha e^{-A-B}
$$

For the null surface, which is associated with $k_{1}$, we have $\Theta=3 \alpha A^{\prime} e^{-A-B}$, while for $k_{2}$ we get $\Theta=3 \beta A^{\prime} e^{-A-B}$.

Then, the counterterm would be

$$
\begin{aligned}
S_{c t}= & -\frac{V_{3}}{4 \pi G_{N}} \int_{\delta}^{r_{m}} d r 3 A^{\prime} e^{3 A} \log \left(3 A^{\prime} e^{-A-B}\right) \\
& -\frac{V_{3}}{8 \pi G_{N}} e^{3 A\left(r_{m}\right)} \log \alpha \beta,
\end{aligned}
$$

where the second term cancels the contribution from the normalization factors of Eq. (4.18).

So, by summing all of the terms and taking the time derivative and also using $\frac{d r_{m}}{d t}=\frac{h\left(r_{m}(t)\right)}{2} e^{A\left(r_{m}(t)\right)-B\left(r_{m}(t)\right)}$, we get

$$
\begin{aligned}
\frac{d S}{d t} & \mid \frac{V_{3}}{\pi G_{N}} \\
= & h\left(r_{m}\right) e^{A\left(r_{m}\right)-B\left(r_{m}\right)}\left(\frac{1}{3} e^{4 A\left(r_{m}\right)+B\left(r_{m}\right)} V\left(r_{m}\right) r^{*}\left(r_{m}\right)\right. \\
& +\frac{3 r_{m}}{8} e^{9 r_{m}^{2}}\left(2+18 r_{m}^{2}-3 \log \left(h\left(r_{m}\right)\right)-\frac{h^{\prime}\left(r_{m}\right)}{6 h\left(r_{m}\right) r_{m}}\right) \\
& \left.-\frac{3}{8} A^{\prime}\left(r_{m}\right) e^{3 A\left(r_{m}\right)} \log \left|3 A^{\prime}\left(r_{m}\right) e^{-A\left(r_{m}\right)-B\left(r_{m}\right)}\right|\right) \\
& +\frac{1}{3} \int_{r_{m}}^{r_{\max }} d z e^{4 A+B} V(z)+\left.\frac{1}{16}\left(e^{-4 A-B} \partial_{r}\left(e^{8 A} h\right)\right)\right|_{r=r_{\max }} .
\end{aligned}
$$

The relationship between $\phi_{m}$ and $t$ can be read numerically from the relation $t=2\left(r^{*}(0)-r^{*}\left(r_{m}\right)\right)$ (note that $\phi=r$ ), and the plots for various $\phi_{H}$ for the different potential models are shown in Fig. 17.

Interestingly, these plots are very similar to the corresponding plots in Ref. [70]. In that work, the confining Einstein-Maxwell-dilaton model was studied, and a 


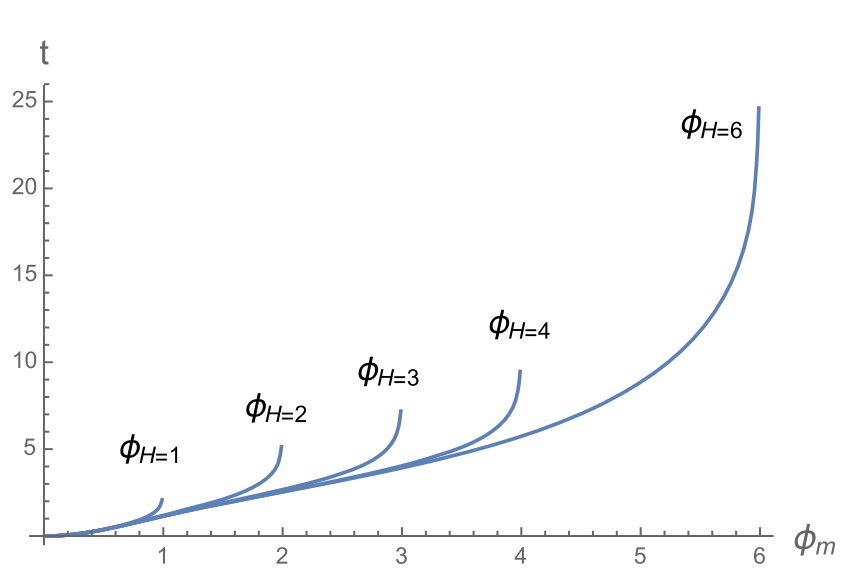

(a) $V_{Q C D}$

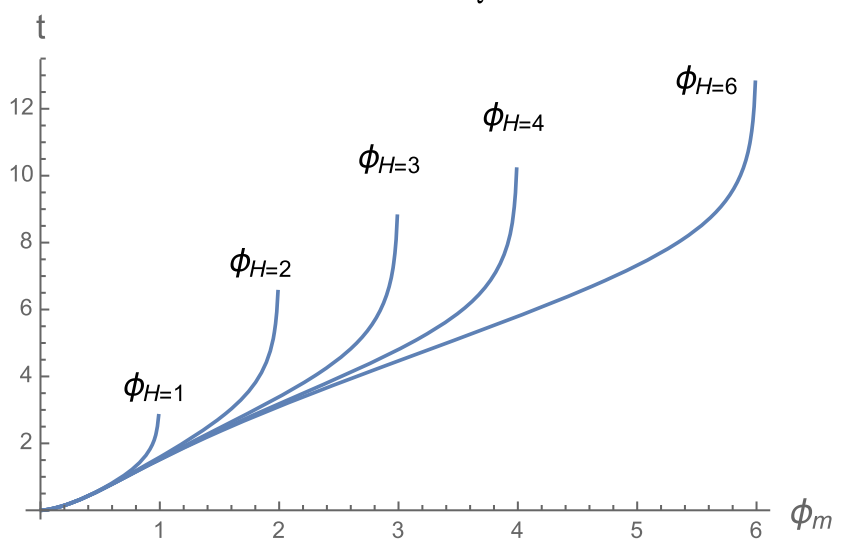

(c) $V_{2}$

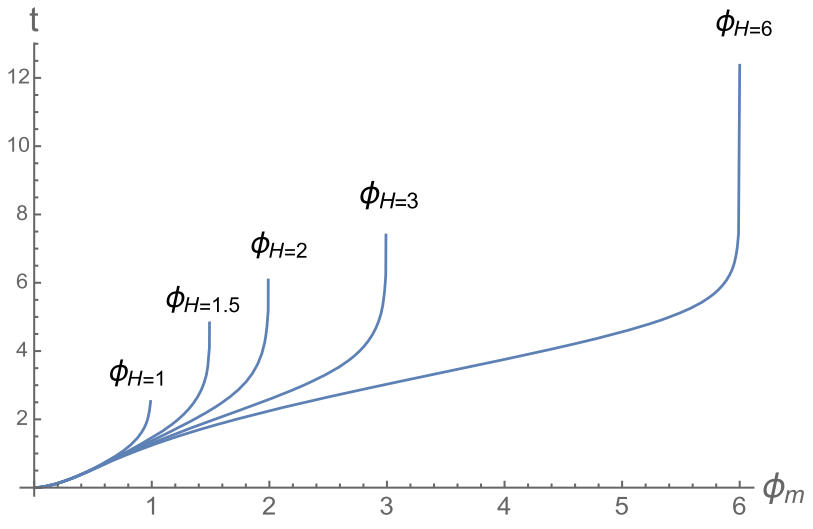

(b) $V_{1}$

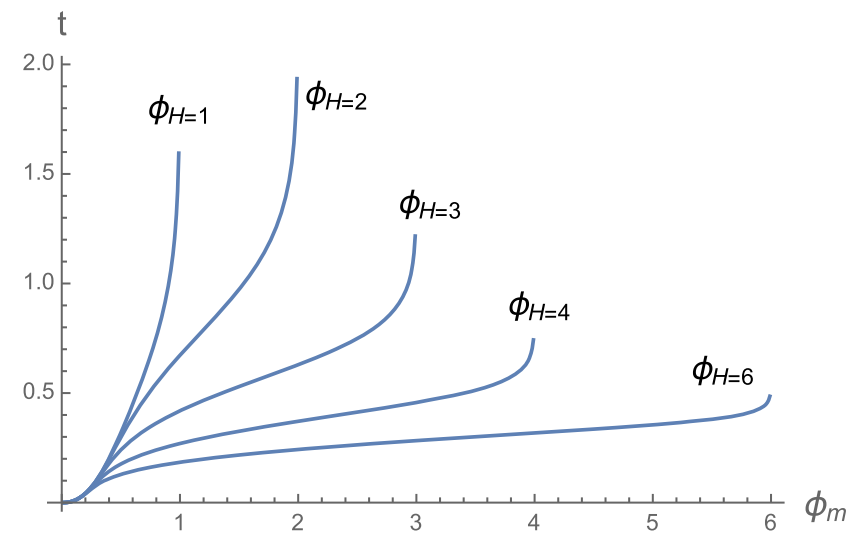

(d) $V_{I H Q C D}$

FIG. 17. The relation between $t$ and $\phi_{m}$.

relationship was found between the length of a strip $\ell$ of a subsystem (which were is used to calculate the entanglement entropy) and the turning point of the minimal area surface $z^{*}$. Note that $z^{*}$ actually corresponds to $\phi_{m}=r_{m}$ in our model. This actually would make sense as $t$ and $l$ are directly connected. Note that when $\phi_{m}$ is smaller than $\phi_{H}$, the relationship between $t$ and $\phi_{m}$ is almost linear, but when $\phi_{m}$ reaches $\phi_{H}, t$ suddenly blows up. These features could constitute a universal behavior of the confining models.

In Fig. 18, we present the full, time-dependent behavior of the complexity growth rate coming from Eq. (4.23) for the case of $V_{\mathrm{OCD}}$. The general behavior is qualitatively very similar for other potential models. From this figure it is obvious that the Lloyd bound would be violated at early times and it would be violated more strongly for black holes with higher temperatures.

However, one might expect that after this sudden increase, the rate of growth would decrease and then saturate at the Lloyd bound. However, with only the terms that we consider here we do not observe such behavior. Therefore, adding some necessary counterterms to Eq. (4.23) would probably solve this issue and, similar to other studies, the Lloyd bound would be saturated from above. We leave finding such terms for these QCD models to future works.

In Fig. 19, we show the behavior of the complexity growth rate $\frac{d C}{d t}$ versus $\phi_{H}$ for late times. One can see that at later times increasing $\phi_{H}$ decreases the complexity growth

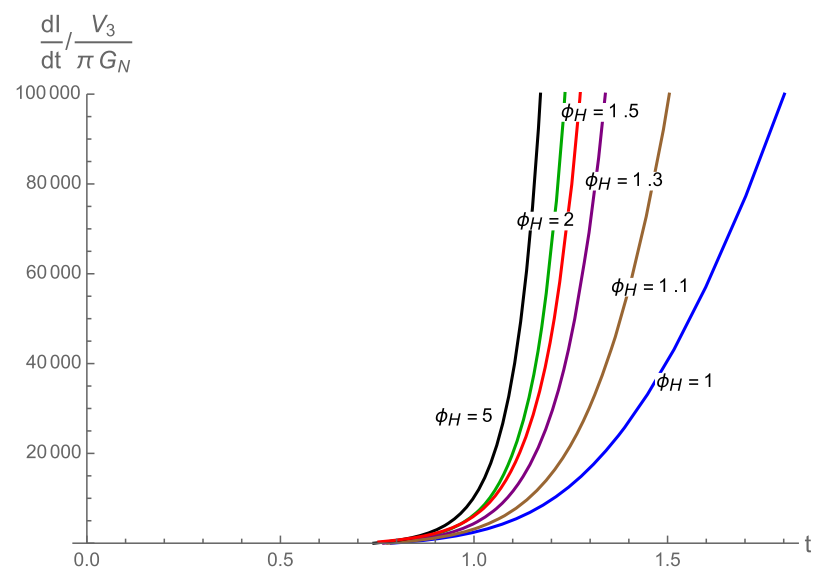

FIG. 18. The complexity growth rate at early times for various black holes in the $V_{\mathrm{QCD}}$ model, with different $\phi_{H}$, corresponding to different temperatures. 


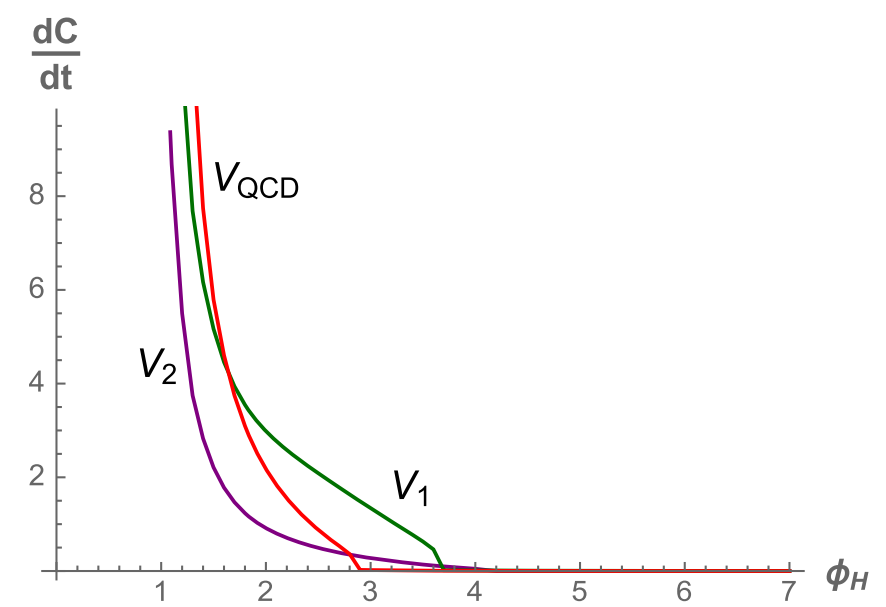

(a) $V_{Q C D, 1,2}$

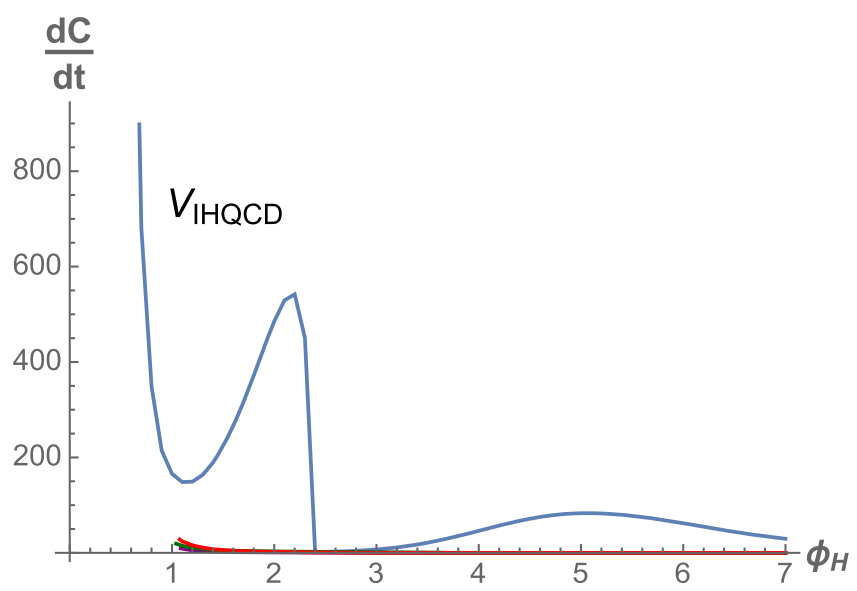

(b) $V_{I H Q C D}$

FIG. 19. The relation between $\frac{d C}{d t}$ and $\phi_{H}$.

rate. Also, the range of complexity growth for $V_{\mathrm{IHQCD}}$ is much larger than the others. The phase transitions of this model for higher $\phi_{H}$ are also evident from the diagram.

By adding gauge fields to the action and using models such as the one in Refs. [71-73] and by doing the same calculations, one can similarly study the full time behavior of the complexity growth rate for the superconducting phases, specifically during the phase transitions [74], which could have many practical applications in quantum information.

We are also interested in studying the effects of hydrodynamics and nonhydrodynamic modes on the complexity growth rate and how these modes affect the different phase transitions during the complexity growth. We discuss this briefly below.

\section{A. $V_{\mathrm{QCD}}$}

The parameters of this confining model have actually been chosen to fit the data for the temperature dependence of the speed of sound, which have been obtained from lattice QCD.

With this potential we observe a crossover behavior at zero baryon charge density. In this case, at lower temperatures near the pseudocritical temperature $T=T_{c}$, there is a rapid change in the large momentum dependence of the imaginary part of the hydrodynamic mode. As momentum increases, the imaginary part flows to minus infinity. As mentioned in Ref. [62], this would point to a novel effect around $T=T_{c}$ in the sound channel where there would be a crossing between the hydrodynamic and nonhydrodynamic modes. It can be seen in Fig. 14(a) that at this point there is actually a sharp decrease in the speed of sound. Then, from Fig. 20(a) one can see that at this point there is a sharp increase in the complexity growth rate.

Also, we note that in all of the figures, at high temperatures, the speed of sound reaches $1 / 3$, which is the expected value for the conformal case or plasmas with a very high $T$.

In the higher-temperature regions, where the results match the conformal case, the nonhydrodynamic modes are actually the most effective ones, while in the lowertemperature regions the hydrodynamic modes play the most important role. Also, it is worth noticing that the hydrodynamic modes (at lower temperature, and around $T=T_{c}$ ) are much more affected by the "ultra locality property" of the system, as mentioned in Ref. [75]. This could actually greatly affect the complexity growth rate of the QCD systems, as we have observed.

\section{B. $\mathbf{V}_{1}$}

This model is actually the most interesting one as it has some distinctive features. The parameters for this model have been constructed in such a way as to generate a firstorder phase transition. In this case one observes an instability or spinodal region for a certain temperature range; this behavior can also be seen in the phase diagrams for the entropy, complexity growth, and especially the speed of sound.

In fact, the first-order phase transition appears in two different scenarios [62]. One is similar to the Hawking-Page scenario where the transition is between a black hole and a thermal gas. The other is between two black holes with different sizes. In this case, for our $V_{1}$ model the first-order phase transition is actually between two black holes [76]. The fact that both of these cases can happen is due to the functional dependence of the dilaton field potential on the radial distance in the deep infrared region. Another distinctive feature is the existence of a nonpropagating sound mode in the very low-temperature range which also shows its effects on the complexity growth rate phase diagram.

However, the most important feature in this model is the presence of the spinodal region which was studied in 


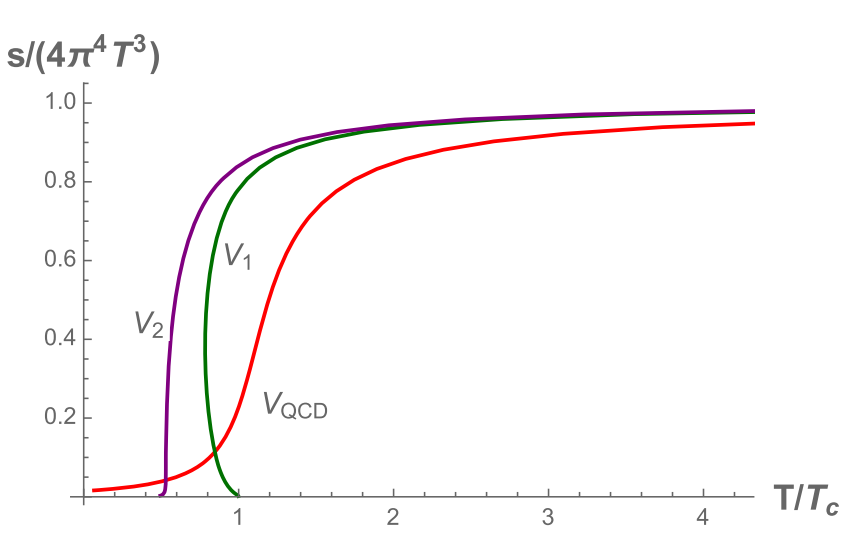

(a) Entropy Phase Diagrams.

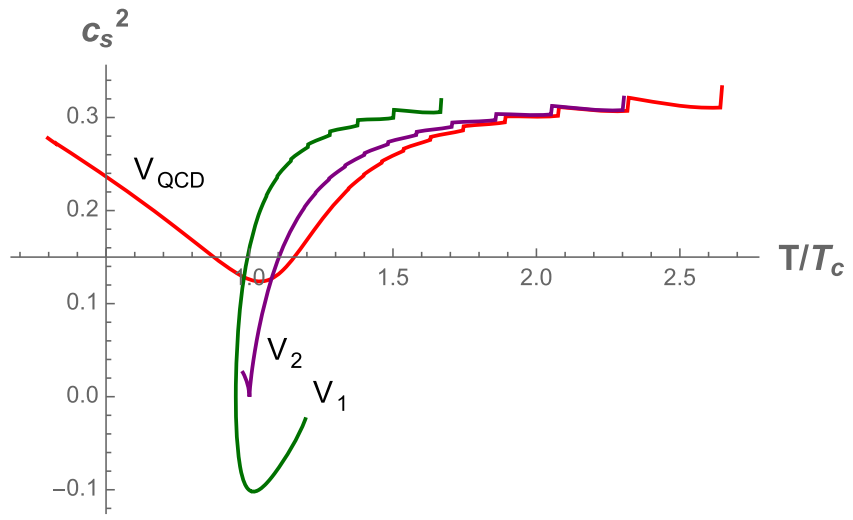

(c) Speed of sound in three models.

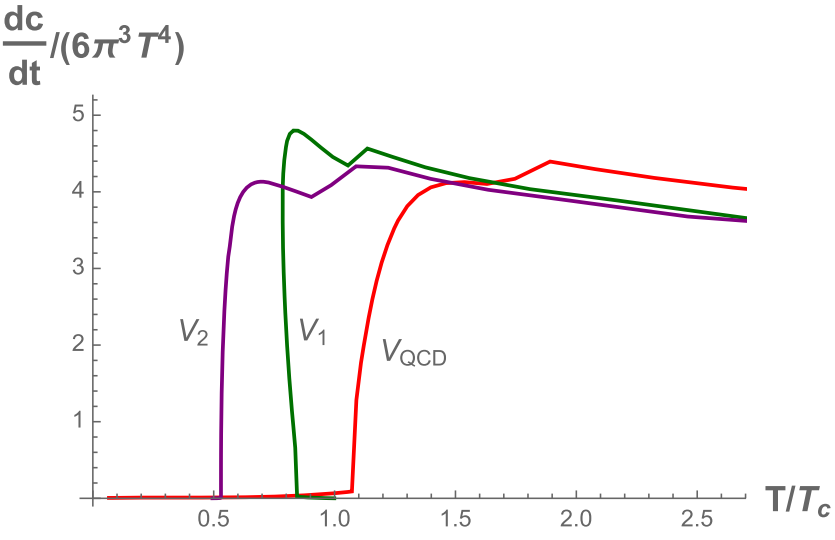

(b) Complexity Phase Diagrams.

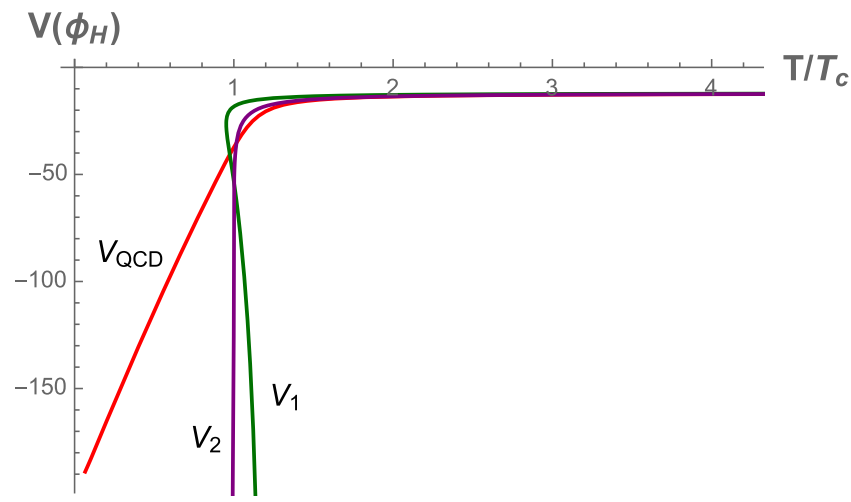

(d) Potentials of the three models.

FIG. 20. Comparing different quantities for three models of QCD.

nuclear physics in the context of spinodal multifragmentation [77]. One can also see from the diagram for the speed of sound (as $\left.c_{s}^{2}<0\right)$ that there exists a dynamical instability during the phase transition which is caused by the bubble formation in this region.

The same kind of behavior has also been observed in gravitational studies of black strings and $p$-branes in the context of Gregory and Laflamme instability [78]. Note that in this case the hydrodynamical modes are purely imaginary and this shows its effect on the complexity growth rate as well. This can be seen from the fact that-unlike the crossover and second-order phase transition where the slopes of $\frac{d C}{d t}$ versus $T / T_{c}$ are positive-in this case the slope of the complexity growth during the phase transition is actually negative. This behavior could be a universal property of complexity for regions with "hydrodynamical instabilities" with a purely imaginary mode.

\section{C. $\mathbf{V}_{2}$}

In this model, at a critical temperature $T=T_{c}$ the speed of sound vanishes, which can be see in Fig. 14(c). Also, near the critical temperature the entropy behaves as

$$
s(T) \sim s_{0}+s_{1}\left(T / T_{c}-1\right)^{1-\alpha},
$$

where, according to Ref. [60], the constant in the power should be $\alpha=2 / 3$. As the complexity growth rate phase diagram is very similar to the entropy diagram, we expect that this relation could approximately describe the complexity growth rate behavior near the critical temperature as well.

Notice that in all of the diagrams for the entropy, complexity growth rate, speed of sound (Fig. 20), and the time dependences of $\phi_{m}$ (Fig. 17) the behavior for the $V_{2}$ case is very similar to the $V_{\mathrm{QCD}}$ case. The main difference is that $V_{2}$ is lagging behind $V_{\mathrm{QCD}}$.

As mentioned in Ref. [62], the generic temperature dependences of the frequencies of the quasinormal modes are also very similar to those of the crossover case. Therefore, this would make all of the corresponding quantities in these two models act similarly around the critical temperature.

Actually, the hydrodynamic description of the system would break down at smaller momenta scales [62]. This would make the critical temperature of $V_{2}$ be lower than in the crossover case. For higher temperatures, all of these 
models including $V_{2}$ would behave the same way and similarly to the conformal case.

In order to get more detailed results about the relationship between the different characteristics of the model and the behavior of the growth of complexity, the numerical data points for the plot of the complexity could be increased. This would then increase the calculation time dramatically.

\section{D. $\mathrm{V}_{\text {IHQCD }}$}

The improved holographic QCD model is very unique and exhibits very different behavior than the other models. It has been constructed in order to better model the dynamical properties of QCD (specifically, asymptotic freedom and color confinement) and to obtain a more realistic value for the bulk viscosity. In this model there is a first-order phase transition, but it is between a black hole and a thermal gas or a vacuum confining geometry, similar to a Hawking-Page phase transition. This fact could actually explain the sharp drop in the phase diagram for the complexity growth rate of this model; see Fig. 15(d).

The exact critical temperature were the first-order phase transition occurs is very difficult to determine for this case, as there are several solutions with instabilities and the temperature of the reference geometry (which is the thermal gas) would be infinite. However, from the diagrams we see the phase transition point has a very distinct behavior since at that point the geometry changes dramatically, which is also apparent in the diagrams for the speed of sound and complexity growth rate.

From these diagrams one can actually detect two characteristic temperatures that are very close to each other. As mentioned in Ref. [62], for temperatures between these two and for low momenta, the lowest-lying excitation modes become purely imaginary which leads to ultralocality violation in this section. This greatly impacts the first phase of the complexity growth rate. Then, we notice that at $T=T_{m}$, for some modes, the hydrodynamic mode and the first nonhydrodynamic mode have the same dispersion relation. At a critical temperature $T_{c}$ (a bit higher than $T_{m}$ ), a transition takes place that substantially changes the geometry. This would cause the rapid increase and then decrease in the plot of the complexity growth rate.

The plot shown in Fig. 14(d) is actually for an unstable smaller black hole. The stable case (which models the behavior of the pure glue system [79]) is not shown here, and we leave it for future studies. The plot of the speed of sound shown here still shows the existence of a spinodal instability and therefore bubble formation. Specifically, for the small black hole region the speed of sound is anomalously large and then becomes superluminal, violating causality. This actually points to a "dynamical" instability, which is different from the "spinodal case" seen before. This difference in behavior could also be detected from the behavior of normal modes [62], and specifically from the complexity growth rate behavior shown in Figs. 13(d), 14(d), and 15(d), and even Fig. 19.

By studying the poles of the Green's functions, the behavior of the hydrodynamic and nonhydrodynamic modes was discussed in more detail in Ref. [62]. It has been shown that the modes are degenerate close to the minimal black hole temperature, and there exists a region of temperatures where the nonhydrodynamical modes are unstable.

In Ref. [62], it was pointed out that a small gap between the degrees of freedom at low momenta could exist. The nonhydrodynamical modes show ultralocal properties as they have weak dependences on the momentum scale. It would be interesting to study the effects of such nonlocal modes on the behavior of the complexity growth rates. Also, a more careful study of the behavior of the complexity growth rate close to the mode gap between the two solutions would be very interesting.

Finally, it is worth noting that as the modes of this system match the results from the holographic dual of superfluid systems [62,80], one would expect that the behavior of the growth of quantum complexity shown in Fig. 15(d) would actually match the behavior of the growth of complexity in superfluid systems, which again could be tested experimentally.

\section{DISCUSSION}

In this work we showed the relationship between different field potentials and the complexity growth rate behavior in several models, including a charged dilaton, and BornInfeld and dyonic black holes. We conjectured that it is a universal feature of complexity that, for potential wells, the complexity growth rate is higher, and for parameters of the model where there is a steep potential barrier the complexity growth rate would decrease to zero. This could indicate that quantum fluctuations such as tunneling are the main source of the complexity growth rate after the thermalization point.

Also, we studied the complexity growth rates during different phase transitions. First, for the dyonic black hole, we studied the full time behavior. Similar to other studies, we found that the Lloyd bound is violated at early times, but then at later times it is saturated from above. Changing the charges of the black hole could reveal a second-order phase transition in the complexity growth rate, which is similar to the phase transition of a van der Waals fluid. For very large charges, the complexity growth rate would diverge (as expected), since in those ranges of charge the black hole would be unstable. Therefore, we found that the complexity growth rate could be a very suitable probe to observe different phase transitions and instabilities.

To further explore this point, we considered the complexity growth rate of AdS soliton black holes, which was studied for the first time in Ref. [31]. We compared the behavior of the complexity growth rates in the AdS soliton 
and pure AdS cases and the potential behavior in the AdS soliton case with tachyonic condensation. We also compared the Schwinger phase diagrams, and again observed strong correlations.

Finally, for the main part of this work we studied the Gubser model of QCD $[59,60]$, and-by tuning the parameters of the potential for the $V_{\mathrm{QCD}}, V_{1}$, and $V_{2}$ cases-we were able to generate the crossover, first-order, and secondorder phase transitions. We also studied the improved holographic QCD potential model $V_{\mathrm{IHQCD}}$, which improves the modeling of the dynamical properties. Solving for the entropies, complexity rates, speeds of sound, and potentials numerically, we observed similarities in their behaviors near the phase transition points in each model. By considering the crossings between hydrodynamic and nonhydrodynamic modes, we then explained different features of the complexity growth rates and phase diagrams.

For the full, time-dependent phase diagrams, however, we showed that additional counterterms in the action for this QCD model would be needed to produce the desired behavior considering the Lloyd bound.

For future works, one could make the argument about the relationship between the complexity growth rate and particle creation and annihilation rates more precise. To do that, one could imagine the Schwinger mechanism as the motion in the space of unitary operators and then, using the intuition from Refs. [27,28], calculate how much pair creation or vacuum quantum fluctuations would increase the distance between the initial state at $t_{0}$ and the state at later times after the creation of only one pair. Holographic methods of considering an open string, writing the NambuGoto action and the induced metric on the string world sheet, and adding proper boundary and joint terms to calculate the action could be used to solve this problem.
The first few steps were taken in the final part of Ref. [34]. The most important step is determining the correct Wheeler-DeWitt patch. By solving these issues and comparing with the results from field theory methods (such as the one in Ref. [30]), one could check whether the creation of these particles would actually move on the optimal path of increasing computational complexity, which would be a very interesting problem.

As a side note, in Refs. [37,58], the decay rate for different backgrounds was found to increase with a magnetic field parallel to the electric field, while it decreases with a magnetic field perpendicular to the electric field, which seems to be a universal feature of the Schwinger effect. One could then check how the complexity growth rates differ in the two setups (i.e., parallel or perpendicular fields). Comparing the results could strengthen our conjecture.

The relationship between the "shear viscosity" of black holes and the complexity growth rate (especially around the critical temperature) could also be studied. This specifically would be an interesting problem with practical applications. For instance, as suggested in Ref. [81], in quarkgluon plasmas there would be a sharp increase in the bulk viscosity near the deconfinement transition, which actually points to the "soft statistical hadronization" of the plasma. So, it would be interesting to study the complexity growth rate at this point and depict the relationship between the complexity and shear or bulk viscosity of black holes and the corresponding QCD phases.

We hope to study these questions in future works.

\section{ACKNOWLEDGMENTS}

I thank Hesam Soltanpanahi and Saeedeh Sadeghian for useful discussions.
[1] S. Ryu and T. Takayanagi, Holographic Derivation of Entanglement Entropy from AdS/CFT, Phys. Rev. Lett. 96, 181602 (2006).

[2] L. Susskind, Computational complexity and black hole horizons, Fortschr. Phys. 64, 24 (2016); 64, 44(A) (2016).

[3] A. R. Brown, D. A. Roberts, L. Susskind, B. Swingle, and Y. Zhao, Complexity, action, and black holes, Phys. Rev. D 93, 086006 (2016).

[4] M. Alishahiha, Holographic complexity, Phys. Rev. D 92 , 126009 (2015).

[5] A. R. Brown, D. A. Roberts, L. Susskind, B. Swingle, and Y. Zhao, Holographic Complexity Equals Bulk Action?, Phys. Rev. Lett. 116, 191301 (2016).

[6] R.-G. Cai, M. Sasaki, and S.-J. Wang, Action growth of charged black holes with a single horizon, Phys. Rev. D 95, 124002 (2017).
[7] A. Bhattacharyya, P. Caputa, S. R. Das, N. Kundu, M. Miyaji, and T. Takayanagi, Path-integral complexity for perturbed CFTs, J. High Energy Phys. 07 (2018) 086.

[8] D. Carmi, S. Chapman, H. Marrochio, R. C. Myers, and S. Sugishita, On the time dependence of holographic complexity, J. High Energy Phys. 11 (2017) 188.

[9] M. Ghodrati, Complexity growth in massive gravity theories, the effects of chirality, and more, Phys. Rev. D 96, 106020 (2017).

[10] R. Auzzi, S. Baiguera, and G. Nardelli, Volume and complexity for warped AdS black holes, J. High Energy Phys. 06 (2018) 063.

[11] R. Auzzi, S. Baiguera, M. Grassi, G. Nardelli, and N. Zenoni, Complexity and action for warped AdS black holes, J. High Energy Phys. 09 (2018) 013. 
[12] R. Abt, J. Erdmenger, M. Gerbershagen, C. M. MelbyThompson, and C. Northe, Holographic subregion complexity from kinematic space, arXiv:1805.10298.

[13] W. Cottrell and M. Montero, Complexity is simple!, J. High Energy Phys. 02 (2018) 039.

[14] C. A. Agn, M. Headrick, and B. Swingle, Subsystem complexity and holography, arXiv:1804.01561.

[15] B. Swingle and Y. Wang, Holographic complexity of Einstein-Maxwell-Dilaton gravity, J. High Energy Phys. 09 (2018) 106.

[16] K. Hashimoto, N. Iizuka, and S. Sugishita, Thoughts on holographic complexity and its basis-dependence, Phys. Rev. D 98, 046002 (2018).

[17] J. L. F. Barbon and J. Martin-Garcia, Terminal holographic complexity, J. High Energy Phys. 06 (2018) 132.

[18] D. Ageev, I. Aref 'eva, A. Bagrov, and M. I. Katsnelson, Holographic local quench and effective complexity, J. High Energy Phys. 08 (2018) 071.

[19] L.-P. Du, S.-F. Wu, and H.-B. Zeng, Holographic complexity of the disk subregion in $(2+1)$-dimensional gapped systems, Phys. Rev. D 98, 066005 (2018).

[20] B. Chen, W.-M. Li, R.-Q. Yang, C.-Y. Zhang, and S.-J. Zhang, Holographic subregion complexity under a thermal quench, J. High Energy Phys. 07 (2018) 034.

[21] A. Ovgn and K. Jusufi, Complexity growth rates for AdS black holes with dyonic/ nonlinear charge/ stringy hair/ topological defects, arXiv:1801.09615.

[22] Z. Fu, A. Maloney, D. Marolf, H. Maxfield, and Z. Wang, Holographic complexity is nonlocal, J. High Energy Phys. 02 (2018) 072.

[23] M. Flory and N. Miekley, Complexity change under conformal transformations in $\mathrm{AdS}_{3} / \mathrm{CFT}_{2}$, arXiv:1806 .08376 .

[24] P. Caputa and J. M. Magan, Quantum computation as gravity, arXiv:1807.04422.

[25] P. Caputa, N. Kundu, M. Miyaji, T. Takayanagi, and K. Watanabe, Liouville action as path-integral complexity: From continuous tensor networks to AdS/CFT, J. High Energy Phys. 11 (2017) 097.

[26] R. Abt, J. Erdmenger, H. Hinrichsen, C. M. MelbyThompson, R. Meyer, C. Northe, and I. A. Reyes, Topological complexity in $\mathrm{AdS}_{3} / \mathrm{CFT}_{2}$, Fortschr. Phys. 66, 1800034 (2018).

[27] R. A. Jefferson and R.C. Myers, Circuit complexity in quantum field theory, J. High Energy Phys. 10 (2017) 107.

[28] S. Chapman, M. P. Heller, H. Marrochio, and F. Pastawski, Towards Complexity for Quantum Field Theory States, Phys. Rev. Lett. 120, 121602 (2018).

[29] R. Khan, C. Krishnan, and S. Sharma, Circuit complexity in fermionic field theory, arXiv:1801.07620.

[30] L. Hackl and R. C. Myers, Circuit complexity for free fermions, J. High Energy Phys. 07 (2018) 139.

[31] A. P. Reynolds and S.F. Ross, Complexity of the AdS soliton, Classical Quantum Gravity 35, 095006 (2018).

[32] M. Guo, J. Hernandez, R. C. Myers, and S.-M. Ruan, Circuit complexity for coherent states, J. High Energy Phys. 10 (2018) 011.

[33] A. Bhattacharyya, A. Shekar, and A. Sinha, Circuit complexity in interacting QFTs and RG flows, J. High Energy Phys. 10 (2018) 140.
[34] J. de Boer, E. Llabrs, J. F. Pedraza, and D. Vegh, Chaotic Strings in AdS/CFT, Phys. Rev. Lett. 120, 201604 (2018).

[35] M. Ghodrati, Hyperscaling violating solution in coupled dilaton-squared curvature gravity, Phys. Rev. D 90, 044055 (2014).

[36] S.-J. Zhang, Complexity and phase transitions in a holographic QCD model, Nucl. Phys. B929, 243 (2018).

[37] M. Ghodrati, Schwinger effect and entanglement entropy in confining geometries, Phys. Rev. D 92, 065015 (2015).

[38] M. Kord Zangeneh, Y. C. Ong, and B. Wang, Entanglement entropy and complexity for one-dimensional holographic superconductors, Phys. Lett. B 771, 235 (2017).

[39] M. Ghodrati and A. Naseh, Phase transitions in BergshoeffHohm-Townsend massive gravity, Classical Quantum Gravity 34, 075009 (2017).

[40] H. A. Camargo, P. Caputa, D. Das, M. P. Heller, and R. Jefferson, Complexity as a novel probe of quantum quenches: Universal scalings and purifications, arXiv: 1807.07075 .

[41] M. Ghodrati, Ph.D. thesis, Michigan University, MCTP, 2016, arXiv:1609.04168.

[42] M. Ghodrati, K. Hajian, and M. R. Setare, Revisiting conserved charges in higher curvature gravitational theories, Eur. Phys. J. C 76, 701 (2016).

[43] M. Alishahiha, A. Faraji Astaneh, M. R. Mohammadi Mozaffar, and A. Mollabashi, Complexity growth with Lifshitz scaling and hyperscaling violation, J. High Energy Phys. 07 (2018) 042.

[44] C. J. Gao and S. N. Zhang, Dilaton black holes in de Sitter or anti-de Sitter universe, Phys. Rev. D 70, 124019 (2004).

[45] Y.-S. An and R.-H. Peng, The effect of dilaton on the holographic complexity growth, Phys. Rev. D 97, 066022 (2018).

[46] A. R. Brown, H. Gharibyan, A. Streicher, L. Susskind, L. Thorlacius, and Y. Zhao, Falling toward charged black holes, arXiv:1804.04156.

[47] S. Dutta, A. Jain, and R. Soni, Dyonic black hole and holography, J. High Energy Phys. 12 (2013) 060.

[48] S. Chapman, H. Marrochio, and R. C. Myers, Holographic complexity in Vaidya spacetimes. Part I, J. High Energy Phys. 06 (2018) 046.

[49] S. Chapman, H. Marrochio, and R. C. Myers, Holographic complexity in Vaidya spacetimes II, J. High Energy Phys. 06 (2018) 114.

[50] A. Chamblin, R. Emparan, C. V. Johnson, and R. C. Myers, Charged AdS black holes and catastrophic holography, Phys. Rev. D 60, 064018 (1999).

[51] H. Xu, Entanglement growth during Van der Waals like phase transition, Phys. Lett. B 772, 517 (2017).

[52] M. A. Nielsen, A geometric approach to quantum circuit lower bounds, arXiv:quant-ph/0502070.

[53] T. Nishioka and T. Takayanagi, AdS bubbles, entropy and closed string tachyons, J. High Energy Phys. 01 (2007) 090.

[54] G. T. Horowitz and E. Silverstein, The inside story: Quasilocal tachyons and black holes, Phys. Rev. D 73, 064016 (2006).

[55] G. T. Horowitz and R. C. Myers, The AdS/CFT correspondence and a new positive energy conjecture for general relativity, Phys. Rev. D 59, 026005 (1998). 
[56] R.-Q. Yang, C. Niu, C.-Y. Zhang, and K.-Y. Kim, Comparison of holographic and field theoretic complexities for time dependent thermofield double states, J. High Energy Phys. 02 (2018) 082.

[57] F. M. Haehl, The Schwarzschild-Black string AdS soliton: Instability and holographic heat transport, Classical Quantum Gravity 30, 055002 (2013).

[58] K. Hashimoto, T. Oka, and A. Sonoda, Electromagnetic instability in holographic QCD, J. High Energy Phys. 06 (2015) 001.

[59] S. S. Gubser, A. Nellore, S. S. Pufu, and F. D. Rocha, Thermodynamics and Bulk Viscosity of Approximate Black Hole Duals to Finite Temperature Quantum Chromodynamics, Phys. Rev. Lett. 101, 131601 (2008).

[60] S. S. Gubser and A. Nellore, Mimicking the QCD equation of state with a dual black hole, Phys. Rev. D 78, 086007 (2008).

[61] R. A. Janik, J. Jankowski, and H. Soltanpanahi, Nonequilibrium Dynamics and Phase Transitions in Holographic Models, Phys. Rev. Lett. 117, 091603 (2016).

[62] R. A. Janik, J. Jankowski, and H. Soltanpanahi, Quasinormal modes and the phase structure of strongly coupled matter, J. High Energy Phys. 06 (2016) 047.

[63] R. A. Janik, J. Jankowski, and H. Soltanpanahi, Real-Time Dynamics and Phase Separation in a Holographic First Order Phase Transition, Phys. Rev. Lett. 119, 261601 (2017).

[64] R. Zllner and B. Kampfer, Phase structures emerging from holography with Einstein gravity-dilaton models at finite temperature, arXiv:1807.04260.

[65] S.-J. Zhang, Holographic entanglement entropy close to crossover/phase transition in strongly coupled systems, Nucl. Phys. B916, 304 (2017).

[66] D. Stanford and L. Susskind, Complexity and shock wave geometries, Phys. Rev. D 90, 126007 (2014).

[67] L. Susskind, Entanglement is not enough, Fortschr. Phys. 64, 49 (2016).
[68] A. Anabalon, T. Andrade, D. Astefanesei, and R. Mann, Universal formula for the holographic speed of sound, Phys. Lett. B 781, 547 (2018).

[69] L. Lehner, R. C. Myers, E. Poisson, and R. D. Sorkin, Gravitational action with null boundaries, Phys. Rev. D 94, 084046 (2016).

[70] D. Dudal and S. Mahapatra, Interplay between the holographic QCD phase diagram and entanglement entropy, J. High Energy Phys. 07 (2018) 120.

[71] S. S. Gubser, Phase transitions near black hole horizons, Classical Quantum Gravity 22, 5121 (2005).

[72] S. S. Gubser, Breaking an Abelian gauge symmetry near a black hole horizon, Phys. Rev. D 78, 065034 (2008).

[73] C. P. Herzog, Lectures on holographic superfluidity and superconductivity, J. Phys. A 42, 343001 (2009).

[74] D. Momeni, S. A. H. Mansoori, and R. Myrzakulov, Holographic complexity in gauge/string superconductors, Phys. Lett. B 756, 354 (2016).

[75] R. A. Janik, G. Plewa, H. Soltanpanahi, and M. Spalinski, Linearized nonequilibrium dynamics in nonconformal plasma, Phys. Rev. D 91, 126013 (2015).

[76] U. Gursoy, E. Kiritsis, L. Mazzanti, and F. Nitti, Holography and thermodynamics of 5D dilaton-gravity, J. High Energy Phys. 05 (2009) 033.

[77] P. Chomaz, M. Colonna, and J. Randrup, Nuclear spinodal fragmentation, Phys. Rep. 389, 263 (2004).

[78] R. Gregory and R. Laflamme, Black Strings and p-Branes are Unstable, Phys. Rev. Lett. 70, 2837 (1993).

[79] G. Boyd, J. Engels, F. Karsch, E. Laermann, C. Legeland, M. Lutgemeier, and B. Petersson, Thermodynamics of SU(3) lattice gauge theory, Nucl. Phys. B469, 419 (1996).

[80] I. Amado, M. Kaminski, and K. Landsteiner, Hydrodynamics of holographic superconductors, J. High Energy Phys. 05 (2009) 021.

[81] F. Karsch, D. Kharzeev, and K. Tuchin, Universal properties of bulk viscosity near the QCD phase transition, Phys. Lett. B 663, 217 (2008). 